\title{
Radiotherapy programs neutrophils to an antitumor phenotype by inducing mesenchymal-epithelial transition
}

\author{
Qiqi Liu ${ }^{1,2,3 \#}$, Yuying Hao ${ }^{1,3,4 \#}$, Rui $\mathrm{Du}^{5}$, Dan Hu${ }^{1,6}$, Jian Xie ${ }^{1,3}$, Jingxin Zhang ${ }^{7}$, Guodong Deng ${ }^{8}$, \\ Ning Liang ${ }^{1,3}$, Tiantian Tian ${ }^{1,3}$, Lukas Käsmann ${ }^{9}$, Dirk Rades ${ }^{10}$, Chai Hong Rim ${ }^{11}$, Pingping Hu ${ }^{1,3}$, \\ Jiandong Zhang ${ }^{1,3}$
}

${ }^{1}$ Department of Oncology, The First Affiliated Hospital of Shandong First Medical University \& Shandong Provincial Qianfoshan Hospital, Jinan, China; ${ }^{2}$ Department of Oncology, Shanghai Medical College, Fudan University, Shanghai, China; ${ }^{3}$ Shandong Lung Cancer Institute, Jinan, China; ${ }^{4}$ Department of Radiation Oncology, Shandong Provincial Qianfoshan Hospital, Cheeloo College of Medicine, Shandong University, Jinan, China; ${ }^{5}$ Division of Oncology, Department of Graduate, Weifang Medical College, Weifang, China; ${ }^{6}$ Department of Physiology, Jeonbuk National University Medical School, Jeonju 54907, Jeollabuk-do, Korea; ${ }^{7}$ Department of Radiation Oncology, Shandong Cancer Hospital Affiliated to Shandong University, Jinan, China; ${ }^{8}$ Department of Chemical Etiology and Carcinogenesis, Cancer Institute, Peking Union Medical College and Chinese Academy of Medical Sciences, Beijing, China; ${ }^{9}$ Department of Radiation Oncology, University Hospital, LMU Munich, Munich, Germany; ${ }^{10}$ Department of Radiation Oncology, University of Lübeck, Lübeck, Germany; ${ }^{11}$ Department of Radiation Oncology, Korea University Ansan Hospital, Ansan, Republic of Korea

Contributions: (I) Conception and design: Q Liu, Y Hao, P Hu; (II) Administrative support: T Tian, P Hu, J Zhang; (III) Provision of study materials or patients: J Zhang, G Deng, N Liang; (IV) Collection and assembly of data: Q Liu, Y Hao, R Du, D Hu, Ji Xie; (V) Data analysis and interpretation: Q Liu, Y Hao, P Hu, T Tian; (VI) Manuscript writing: All authors; (VII) Final approval of manuscript: All authors.

\#These authors contributed equally to this work.

Correspondence to: Pingping Hu; Jiandong Zhang. Department of Oncology, The First Affiliated Hospital of Shandong First Medical University \& Shandong Provincial Qianfoshan Hospital, 16766 Jingshi Road, Jinan 250014, China. Email: hupingping0915@126.com; Jiandongzhang165@163.com.

Background: Neutrophils can play a pro-tumor or anti-tumor role depending on the tumor microenvironment. The effects of concurrent treatment with granulocyte colony-stimulating factor (G-CSF) and radiotherapy (RT) on neutrophils have not yet to be described.

Methods: Hypofractionated radiation of $8 \mathrm{~Gy} \times 3$ fractions was administered with or without recombinant G-CSF to Lewis lung carcinoma tumor-bearing C57BL/6 model mice. The activation status of cytotoxic $\mathrm{T}$ cells in the mice was measured, along with the levels of tumor-associated neutrophils, cytotoxic $\mathrm{T}$ cells, and Treg cells. Tumor growth, survival, cytokine expression, and signaling pathways underlying antitumor effects of tumor-associated neutrophils after treatment were also studied. To ascertain the effects of concurrent RT and G-CSF on tumor-associated neutrophils, neutrophil depletion was performed.

Results: RT affected early neutrophil infiltration, which is the first-line immune response. Subsequently, enhanced accumulation of lymphocytes, particularly CD8 cytotoxic T cells, was observed. Notably, lymphocytic infiltration was inhibited by neutrophil depletion but enhanced by G-CSF treatment. RT generated persistent DNA damage, as evidenced by an accumulation of phosphorylation of histone H2AX $(\gamma \mathrm{H} 2 \mathrm{AX})$, and subsequently triggered inflammatory chemokine secretion. The chemokines CXCL1, CXCL2, and CCL5 were upregulated in both radiation-treated cells and the corresponding supernatants. Neutrophils that were newly recruited after RT improved radiosensitivity by inhibiting epithelial-mesenchymal transition via the reactive oxygen species-mediated PI3K/Akt/Snail signaling pathway, and G-CSF treatment enhanced this effect.

Conclusions: The results of this study suggest that RT activates neutrophil recruitment and polarizes newly recruited neutrophils toward an antitumor phenotype, which is enhanced by the concurrent administration of G-CSF. Mesenchymal-epithelial transition induced by reactive oxygen species accumulation plays a major role in this process. Thus, the polarization of tumor-associated neutrophils might play a role in future cancer immunotherapies. 
Keywords: Radiation therapy; tumor-associated neutrophils; mesenchymal-epithelial transition; radiosensitivity; immunotherapy

Submitted Oct 19, 2020. Accepted for publication Mar 25, 2021.

doi: $10.21037 /$ tlcr-21-152

View this article at: http://dx.doi.org/10.21037/tlcr-21-152

\section{Introduction}

External radiotherapy (RT) has been a main modality of definitive treatment for locally advanced lung cancers. The indications for early lung cancer are also increasing using the latest treatment techniques such as stereotactic radiotherapy (1). However, in the case of locally advanced lung cancer (unresectable stage III), the 3-year survival rate is less than a quarter in those receiving $\mathrm{RT}$ and chemotherapy as initial treatment (2). Therefore, there is an urgent need for a complementing agent to increase the efficiency of radiotherapy.

The effect of radiation as an anticancer treatment relies mainly on the direct DNA damage resulting in DNA double-strand breaks (DSBs) which activate different cellular DNA damage responses (DDRs) such as DNA damage sensing, cell cycle arrest, DNA repair and activation of cellular death pathways (3). On the other hand, ionizing radiation causes direct cytotoxic effects such as tumor cell necrosis which releases tumor-specific antigens, including adenosine triphosphate, heat shock proteins and high mobility group box 1 protein, eliciting an antitumor immune response (4). Increasing evidence suggests that RT affects the tumor microenvironment (TME) by stimulating many tumor-promoting factors, and plays a key role in immune escape, angiogenesis, and tumor promotion in several cancers, including lung cancer $(5,6)$.

The TME is complex and continuously evolving, which is crucial for tumor development and progression. The TME comprises tumor cells, stromal cells, and immune cells that secrete a wide variety of cytokines, chemokines, and growth factors, which act as a paracrine fashion to affect the nearby tumor cells. Some of these paracrine signals induce an epithelial-mesenchymal transition (EMT), characterized by the morphological changes of the cells lose their epithelial appearance and acquire markers of mesenchymal cells. This process is reversible, and mesenchymal cells revert into epithelial cells during the process of mesenchymal-epithelial transition (MET) (7). The crosstalk between tumor and immune cells is required for tumor progression and metastasis (8). In cancer, neutrophils have emerged as an important component of the TME. Recently, the role of tumor-associated neutrophils (TANs) in the development of cancer has received considerable attention. Similar to macrophages, TANs display an N1 phenotype with anti-tumor effects and an N2 phenotype with pro-tumor effects while the phenotype transition depends on the TME (9). TANs can be a component of tumor-promoting inflammation by promoting angiogenesis, immunosuppression, remodeling of the extracellular matrix, and metastasis. On the other hand, TANs exhibit antitumor effects by direct killing of tumor cells, and interacting with other components of the immune system (10). Transforming growth factor (TGF)- $\beta 1$ promotes the polarization of TANs to the $\mathrm{N} 2$ phenotype, whereas blocking TGF- $\beta 1$ results in a shift toward the $\mathrm{N} 1$ phenotype (9). A low expression of interferon (IFN)- $\beta$ causes pro-tumor $\mathrm{N} 2$ neutrophils to accumulate in tumors, which has a tumor-promoting effect (11). However, subsets of neutrophils are defined by morphology, and the conversion programmed by the TME cannot be detected due to a lack of specific markers. Garg et al. discovered that RT recruited neutrophils to tumor sites (12); however, the potential effects and underlying mechanisms of RTrecruited neutrophils have not yet been characterized.

$\mathrm{G}-\mathrm{CSF}$ is indispensable to progenitor differentiation and the transformation of granulocytic lineages, including neutrophils, eosinophils, and basophils. In addition to its hematopoietic properties, G-CSF also mobilizes progenitors, hematopoietic stem cells, and mature cells, especially neutrophils, into the blood circulation (13). Thus, the effects of RT-recruited neutrophils on tumors can be enhanced by concurrent G-CSF treatment.

We hypothesized that neutrophils may play a pivotal role in the TME after concurrent treatment with G-CSF and RT. Herein, we established a mouse model to elucidate the potential effects of neutrophils recruited to tumors by RT and G-CSF, with the aim of identifying a potential immunotherapeutic target for the treatment of cancer.

We present the following article in accordance with the ARRIVE reporting checklist (available at http://dx.doi. 
org/10.21037/tlcr-21-152).

\section{Methods}

\section{Animals}

Female C57BL/6 mice (Beijing Vital River Laboratory Animal Technology; Beijing, China), aged 6-8 weeks $(18-22 \mathrm{~g})$, were housed individually under specific pathogen-free conditions with adequate food and water and kept under a 12 -hour light-dark cycle at $22-25^{\circ} \mathrm{C}$. All animal experiments conducted were reviewed and approved by the Institutional Animal Care and Use Committee of Shandong Provincial Qianfoshan Hospital (no. 2016-S017). Experiments were conducted in accordance with the Guide for Care and Use of Laboratory Animals (NIH publication no. 85-23, revised 1996). After a 1-week acclimation period, the mice were given a subcutaneous injection of $1 \times 10^{6}$ C57BL/6-derived Lewis lung carcinoma (LLC) cells into the right flank. When the tumors reached $6-8 \mathrm{~mm}$ in diameter, the animals were subjected to focal radiation and other treatments. All mice were euthanized by cervical dislocation, and their tumors, spleens and lymph nodes were harvested for subsequent experiments. The antitumor effects of RT and G-CSF were determined by assessing the tumor volume, which was calculated twice a week using the following formula: tumor volume $=0.5 \times$ length $\times$ width $^{2}$. The tumor weights were also measured after dissection. The mice included in the survival analysis study were allowed to die naturally. Survival data were collected as the percentages of surviving mice each day after LLC inoculation. However, if weight loss exceeded $20 \%$ or a mouse became immobile and unable to intake food, euthanasia was performed. Nontumor-related deaths were also excluded from the death records in the present analysis.

\section{Mouse treatment}

C57BL/6 mice were anesthetized with $300 \mathrm{mg} / \mathrm{kg} 10 \%$ chloral hydrate (Qilu Pharmaceutical; Jinan, Shandong, China) and placed on a tray with the cancer lesions positioned in the field of radiation. On days 1, 3, and 5, a dose of 8 Gy was administered at $6 \mathrm{~Gy} / \mathrm{min}$ with 6-MV high-energy photon rays (Varian TrueBeam Linacs, Varian Medical Systems Inc., Palo Alto, CA, USA). To minimize whole-body exposure, the radiation field focused on the tumors was set as $10 \times 10 \mathrm{~mm}^{2}$. Recombinant mouse G-CSF $(150 \mu \mathrm{g} / \mathrm{kg}$, Novus, Novus Biological, Littleton, CO, USA) was subcutaneously injected in proximity to the tumor immediately after each application of radiation. For neutrophil depletion, the mice were intratumorally injected with $300 \mu \mathrm{g}$ anti-Ly6G monoclonal antibody (mAb in $100 \mu \mathrm{L}$ PBS, clone $1 \mathrm{~A} 8$, BioXcell Life Sciences; West Lebanon, NH, USA) 1 day before radiation, and then 3 times a week. The control mice received the equivalent volume of saline. The dose of G-CSF and anti-Ly6G monoclonal antibody was determined based on the previous published results $(14,15)$.

The mice were randomly divided into different experimental and control groups as follows: (I) control group, (II) RT and anti-Ly6G mAb group, (III) anti-Ly6G mAb group, (IV) RT group, (V) G-CSF group, and (VI) RT and G-CSF group. The Ly6G group was used to verify the effectiveness of neutrophil depletion as well as the roles of neutrophils in antitumor effect independently of radiotherapy. Further, to investigate the roles of individual chemokines in neutrophil chemotaxis, mouse cytokine array analysis (R\&D Systems; Abingdon, UK) was conducted, in which CXCL1, CXCL2, and CCL-5-blocking antibodies (used at $50 \mathrm{ng} /$ body, 1:1:1 proportion, R\&D Systems) were applied 3 times at 1 -week to assess the roles of these cytokines. A time-line diagram for study design was showed in Figure S1.

\section{Cell culture}

Cancer cells were cultured by Roswell Park Memorial Institute (RPMI) 1640 medium (Gibco, USA) in tissue culture flasks (Corning, USA) at $37{ }^{\circ} \mathrm{C}$ in a humidified incubator containing $95 \%$ air and $5 \% \mathrm{CO}_{2}$. The complete 1640 medium contained $10 \%$ heat-inactivated fetal bovine serum (FBS, Gibco) and 1\% penicillin-streptomycin (HyClone; GE Healthcare, Little Chalfont, UK) The culture medium was replaced every 2 days and examined periodically for mycoplasma contamination. Phosphatebuffered saline (PBS) was obtained from Sigma-Aldrich St. Louis, MO, USA). Tumor cells LLC cells were supplied by the Shandong Academy of Medical Sciences.

\section{Preparation of single-cell suspensions}

The spleens, draining lymph nodes, and tumor tissues were harvested from the LLC-bearing mice 3 days after the final irradiation. Cell suspensions were obtained by squeezing and homogenizing the spleens and tumor-draining lymph nodes with a tissue homogenizer with PBS. Then, the tumor tissues were minced with scissors and dissociated in tubes containing $1 \mathrm{mg} / \mathrm{mL}$ collagenase IV (Solarbio; Beijing, 
China). The reactions were terminated with 10\% FBS. Subsequently, single cells were obtained by passing through a 0.45-mm nylon mesh (BD Pharmingen, San Diego, CA, USA). After centrifugation at 1,200 rpm for 5 minutes, the supernatant was discarded and resuspended in erythrocyte lysis buffer (Solarbio), and then put on ice for 15 minutes. Following that, centrifugation washes were carried out at 2,000 rpm for 10 minutes. The single-cell suspensions were then washed three times with normal saline and cultured in RPMI 1640 at $37^{\circ} \mathrm{C}$ for further analysis.

\section{Immunobistochemistry}

Immunohistochemistry (IHC) was performed employing the streptavidin-peroxidase method. Briefly, formalinfixed, and paraffin-embedded tumor tissues were sliced into 4- $\mu$ m-thick sections. After dewaxing, rehydration, and citrate antigen retrieval, primary antibodies were applied to the sections on slides and incubated overnight at $4{ }^{\circ} \mathrm{C}$. After that, the slides were incubated with secondary antibodies (SP-9000, ZSGB-BIO, China) at $37^{\circ} \mathrm{C}$. CD8, CD11b, vimentin, and $\mathrm{E}$-cadherin staining was performed with anti-CD8 (BOSTER, Wuhan, China; 1:400), anti-CD11b (Abcam; 1:200), anti-vimentin (Cell Signaling Technology, CST, Boston, MA, USA; 1:300), and anti-E-cadherin (CST; 1:300), respectively. Then, the slides were developed with 3,3'-diaminobenzidine (DAB) and counterstained with hematoxylin. The section without primary antibodies was used as the negative controls. The staining was scored as follows: 0 , no staining; $1,<50 \%$ cells weakly stained; 2 , $\geq 50 \%$ cells weakly stained; $3,<50 \%$ cells strongly stained; and $4, \geq 50 \%$ cells strongly stained. The IHC staining was scored independently in double-blindfolded manners by two researchers. Images of three representative fields were captured with an Olympus BX53 microscope (Olympus, Tokyo, Japan) at $200 \times$ or $400 \times$ magnification.

\section{Flow cytometry}

The proportions or numbers of cytotoxic $\mathrm{T}$ lymphocytes (CTLs, CD8+), TANs (CD11b+Ly6G+), and regulatory T cells (Treg cells, CD4+CD25+FoxP3+) were evaluated on day 3 after antitumor treatment. Neutrophils were stained with two different combinations of cell surface markers, CD11b-APC (BioLegend, San Diego, CA, USA) and Ly6G-PE (clone GR-1, eBioscience, San Diego, CA, USA). Flow cytometry (FC) analysis of CD8 on the surface of suspended single cells was performed with anti-CD8 monoclonal antibody (FITC, eBioscience). Granzyme was stained with anti-granzyme B antibody (Alexa Fluor 647, Biolegend). A mouse regulatory $\mathrm{T}$ cell staining kit (eBioscience) was used to detect Treg cells in accordance with the manufacturer's protocol. Briefly, after incubation with CD4-FITC $\mathrm{mAb}$ and CD25-APC mAb for 30 minutes in the dark at room temperature, cells were fixed and permeabilized with fixation/permeabilization solution (eBioscience). Subsequently, CD4/CD25 double-stained samples were incubated with anti-FoxP3-PE antibody (eBioscience) for further 30 minutes in the dark at $4{ }^{\circ} \mathrm{C}$. All antibodies were used at 1:20 dilution in PBS and added to $10^{6}$ cells within 15 minutes at room temperature. The cells were washed, fixed in 1\% paraformaldehyde fixative (Solarbio), and resuspended in PBS before analysis with a fluorescence-activated cell sorter (FACS Canto II, BD Pharmingen). A minimum of 100,000 cells were counted per sample, and the gating strategy and representative flow cytometry are shown in Figure S2. Results were analyzed with FlowJo Software (Tree Star, Ashland, OR, USA).

\section{In vitro experiments}

All LLC cells were cultured according to standard protocols as described above. First, the LLC cells were incubated in 6-well culture plates and RT was administered at a dose of 8 Gy with 6-MV high-energy photon rays on days 1,3 , and 5. The chemokines released were assessed 1 day after the radiation treatment. A Mouse Cytokine Array Panel A (R\&D Systems) was employed to assess the cell supernatants for 40 mouse cytokines with inflammatory, homeostatic, or dual functions. To confirm the effects of specific cytokines on tumors, the unchanged cell supernatants obtained through centrifugation at 2,000 rpm for 10 minutes at 12 hours and at $1,2,3,5$, and 7 days after irradiation were considered as conditioned media. These supernatants were evaluated using enzyme-linked immunosorbent assay (ELISA) kits for CXCL1, CXCL2, and CCL5 purchased from CUSABIO (Wuhan Huamei Biotech Co., Ltd, Wuhan, China) in accordance with the manufacturer's instructions. The corresponding cells were used for western blotting and quantitative real-time PCR (RT-qPCR) experiments.

\section{Measurement and depletion of reactive oxygen species}

The concentration of intracellular reactive oxygen species (ROS) was evaluated with 2',7'-dichlorodihydrofluorescein diacetate substrate (DCFH-DA; Sigma, Munich, Germany). 
After isolation through density gradient centrifugation according to protocols from Percoll PLUS/Percoll (SigmaAldrich), neutrophil suspensions were incubated with $10 \mu \mathrm{mol} / \mathrm{L}$ DCFH-DA in RPMI 1640 for 30 minutes at $37^{\circ} \mathrm{C}$, and this step was followed by washing neutrophil suspensions with RPMI 1640. In the ROS depletion experiment, the mice were intragastrically gavaged with $\mathrm{N}$-acetylcysteine (NAC, Beyotime, Haimen, China; $200 \mathrm{mg} / \mathrm{kg}$ ) 30 minutes before the first irradiation and 2 days after the last irradiation. The fluorescence data were acquired at 488 and $525 \mathrm{~nm}$ for excitation and emission, respectively, using a FACS Canto II cytometer, and analyzed with FlowJo Software (Tree Star).

\section{Western blotting}

Western blotting was performed according to standard protocols. Cell lysate was prepared in RIPA lysis buffer (Beyotime, Haimen, China), separated through electrophoresis and then transferred onto polyvinylidene difluoride (PVDF) membrane (Solarbio). The membrane was blocked with $5 \%$ nonfat milk in Tris-buffered saline containing $0.1 \%$ Tween-20 for 60 minutes at $37{ }^{\circ} \mathrm{C}$. Following that, membranes were incubated with the primary antibody to E-cadherin (CST; 1:1,000), vimentin (CST; 1:1,000), Snail (CST; 1:1,000), PI3K (Proteintech, Chicago, IN, USA; 1:1,000), pPI3K (CST; 1:1,000), AKT (Proteintech; 1:1,000), pAKT (Proteintech; 1:1,000), and $\gamma$-H2AX (Upstate, Billerica, MA, USA; 1:1,000) overnight at $4{ }^{\circ} \mathrm{C}$. Next, the membranes were then incubated with the appropriate secondary antibodies conjugated to horseradish peroxidase (Proteintech, $1: 10,000$ ) at $37^{\circ} \mathrm{C}$ for 1 hour. The signals from the immunoreactive bands were visualized with enhanced chemiluminescence (ECL) substrate (Millipore, Billerica, MA, USA). Glyceraldehyde 3-phosphate dehydrogenase (GAPDH, Proteintech, 1:1,000) served as the internal control for normalization. The band densities were quantified using ImageJ software (version 1.62; National Institutes of Health, Bethesda, MD, USA). The antibodies used are described in Table S1.

\section{$R T-q P C R$}

Total RNA was extracted with TRIzol (Invitrogen, Grand Island, NY, USA) from irradiation-treated LLC cells. Then, cDNA was synthesized using a ReverTra Ace qPCR RT Kit (Toyobo, Osaka, Japan) According to the manufacturer's protocol. RT-qPCR was conducted using TransStart Green
qPCR SuperMix (TaKaRa Bio Inc., Dalian, China) on an ABI ViiA7 Dx instrument (Applied Biosystems; Thermo Fisher Scientific, Waltham, MA, USA) to determine the messenger RNA (mRNA) expression levels of the target genes. The samples were amplified in three independent experiments.

The primer sequences for each gene were as follows: CXCL1, forward: 5'-TGGCTGGGATTCACCTCAA-3', reverse: 5'-GGCTATGACTTCGGTTTGG-3'; CXCL2, forward: 5'-TCAAGAATGGGCGGAAAG-3', reverse: 5'-CTTCAGGGTCAAGGCAAAC-3'; CCL5, forward: 5'-AAGATCTCTGCAGCTGCCCTCACCA-3', reverse: 5'-TGAGGGCAGCTGCAGAGATCTTCAT-3'; and GAPDH, forward: 5'-TGTTTCCTCGTCCCGTAG-3', reverse: 5 '- CAATCTCCACTTTGCCACT-3. The $2^{-\Delta \Delta C t}$ method was used to calculate relative gene expressions, with GAPDH used as the reference gene for normalization.

\section{SsRNA-seq library construction}

Total RNA was isolated using RNeasy mini kit (Qiagen, Germany). According to the TruSeq ${ }^{\mathrm{TM}}$ RNA sample preparation guide, use the TruSeq ${ }^{\mathrm{TM}}$ RNA sample preparation kit (Illumina, USA) to synthesize the paired end library. Simply put, use poly-T oligomagnetic beads to purify poly-A-containing mRNA molecules. After purification, it was cleaved into small fragments with divalent cations at $94^{\circ} \mathrm{C}$, and the cleaved RNA fragments were copied into the first strand cDNA with reverse transcriptase and random primers. This is the second subsequent cDNA synthesis using DNA polymerase I and RNase $\mathrm{H}$. These cDNA fragments then go through an end repair process, adding a single'a' base, and then connecting the adaptor. Then the product was purified and enriched by PCR to form the final cDNA library. The purified library was quantified by Qubit ${ }^{\circledR} 2.0$ Fluorometer (Life Technologies, USA) and verified by Agilent 2100 Bioanalyzer (Agilent Technologies, USA) to confirm the insert size and calculate the molarity. cBot diluted the library to $10 \mathrm{pm}$ to generate clusters, which were then sequenced on Illumina NovaSeq 6000 (Illumina, USA). Library construction and sequencing work done by the Shanghai Branch of the Group Company.

\section{Statistical analysis}

Differences between groups were evaluated by performing the Unpaired T test. Each experiment was performed 
three times with 3-4 mice per group. The Kaplan-Meier method was used to calculate the cumulative survival time, and differences in survival were analyzed with the logrank test. Overall survival (OS) was defined as the interval between the start of the treatment and death. Unpaired $t$-test was used to compare tumor growth between the groups. Data are presented as the mean $\pm \mathrm{SD}$ (standard deviation). All data were analyzed with two-tailed tests, with $\mathrm{P}<0.05$ considered to show statistical significance. All statistical analyses were performed with SPSS statistical software V19.0 (SPSS Inc., Chicago, IL, USA).

\section{Results}

\section{$R T$-recruited neutrophils improve radiosensitivity and survival}

The recruitment of neutrophils and tumor-specific CTLs in tumors over time following irradiation was investigated (Figure 1A). The number of recruited neutrophils reached a peak at 24 hours post irradiation, after which the number of CTLs began to increase slowly, peaking at 60 hours after irradiation. These results demonstrated that RT leads to recruitment of neutrophils as first-line immune responders. Subsequently, radiosensitivity in LLC-bearing mice was assessed according to tumor volume variation data (Figure 1B). Compared with that in the control group, the rate of tumor growth was significantly impeded in the RTtreated mice $(\mathrm{P}=0.008)$, particularly in those administered concurrent G-CSF and RT $(\mathrm{P}<0.001)$. Further, the OS of mice in different groups was evaluated. The survival of mice that received RT or a combination of RT with G-CSF was significantly longer than that of the control group. Moreover, these results were consistent with our hypothesis that RT-mediated recruitment of neutrophils produces an antitumor effect and thereby prolongs the survival of LLC tumor-bearing mice (Figure 1C).

\section{RT triggers cytokine secretion via persistent DNA damage}

LLC cells were irradiated with 8 Gy three times, $\gamma \mathrm{H} 2 \mathrm{AX}$ expression in the cells was evaluated one day after irradiation. Western blotting showed that the levels of $\gamma \mathrm{H} 2 \mathrm{AX}$, a marker of persistent DNA damage, were significantly increased in irradiated LLC cells $(\mathrm{P}=0.016$, Figure 1D), which confirmed the development of persistent chromatin lesions. Earlier studies showed that cytokine secretion occurs after the establishment of persistent DNA damage signaling (16). Untreated and RT-treated LLC cells were analyzed for the presence of 40 cytokines with a Mouse Cytokine Array Panel A. The RT-treated cells exhibited an increased release of specific cytokines CXCL1, CXCL2, and CCL5 (Figure 1E). Next, RT-qPCR and ELISA were performed on irradiated LLC cells and the corresponding cell supernatants. RT was found to regulate the expression of CXCL1, CXCL2, and CCL5 in a time-dependent manner, and CXCL2 and CCL5 began to decrease 7 days after irradiation (Figure $1 F, G$ ). A heatmap showing the quantification of cytokine expression is depicted in Figure 1H,I.

\section{$R T$ recruitment of neutrophils is dependent on chemokines}

To verify the roles of CXCL1, CXCL2, and CCL5 in the recruitment of TANs, mice were injected with the corresponding antibodies. The simultaneous blockade of CXCL1, CXCL2, and CCL5 largely impeded RT-mediated neutrophil recruitment, as compared with the results in the control group $(\mathrm{P}<0.001)$. Moreover, TAN recruitment was also downregulated following the administration of each neutralizing antibody, albeit to a lesser extent than when all three cytokines were simultaneously blocked $(\mathrm{P}=0.008$, 0.012 , and 0.023 for CXCL1, CXCL2, and CCL5, respectively; Figure 17). In this study, neutrophils were discovered to be first responders and to carry out important functions.

\section{RT leads to recruitment of neutrophils as first-line immune responders}

As shown previous, we discovered that RT leads to recruitment of neutrophils as first-line immune responders. The number of recruited neutrophils reached a peak at 24 hours post irradiation, after which the number of CTLs began to increase slowly, peaking at 60 hours after irradiation. Subsequently, CD11b+Ly6G+TAN infiltration in the tumor by IHC (Figure $2 A$ ) and TAN infiltration in the tumors and spleens of the mice by FC (Figure $2 B$ ) on day 3 after local irradiation and was investigated. The number of TANs was significantly higher in the irradiated mice, especially in the group receiving G-CSF administration, compared to the control mice. The subsequent intratumoral administration of anti-Ly6G antibody resulted in almost $60 \%$ and $80 \%$ neutrophil depletion in the tumors and spleens of irradiated mice, respectively (all $\mathrm{P}<0.05$ ). This successful depletion of neutrophils in the mouse models 

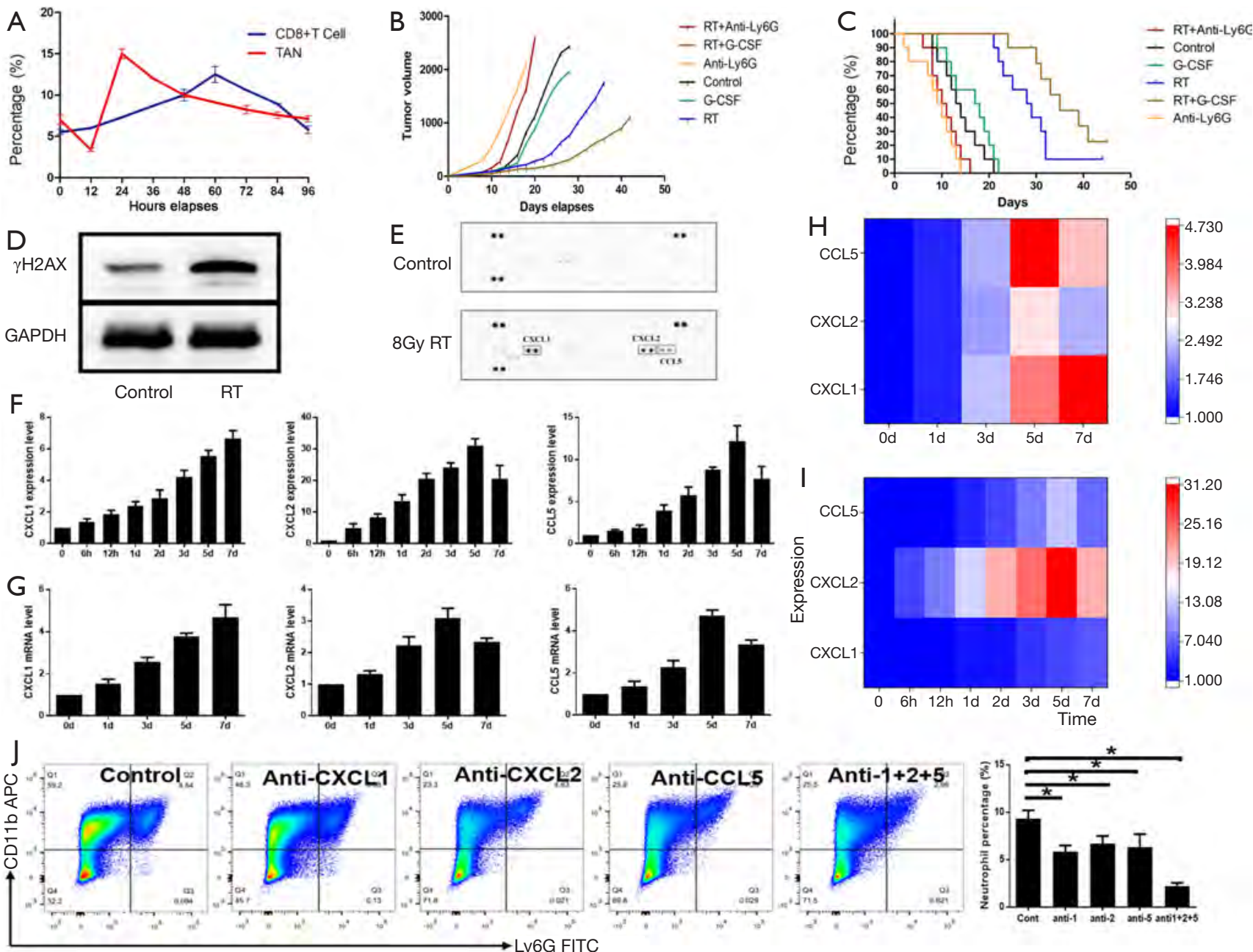

Figure 1 RT leads to recruitment of neutrophils via DSB-induced cytokine secretion. Changes in the percentage of TANs and cytotoxic lymphocyte $\mathrm{T}$ cells in the tumor tissues over time after irradiation were analyzed by flow cytometry (A). Tumor volumes (B) and survival (C) of LLC tumor-bearing C57BL/6 mice are shown for the control group (black), anti-Ly6G group (orange), RT + anti-Ly6G group (red), RT group (blue), G-CSF group (green), and RT + G-CSF group (purple). LLC cells were irradiated with 8 Gy 3 times and analyzed 1 days later for $\gamma-\mathrm{H} 2 \mathrm{AX}(\mathrm{D})$. The cytokine profiles of LLC cells after irradiation were analyzed with a mouse cytokine array panel (E). The expression levels of CXCL1, CXCL2, and CCL5 in supernatants (F,H), and messenger RNA (mRNA) levels in irradiated LLC cells (G,I) are shown in histograms and heatmaps. TANs in the gated population of lymphocytes and granulocytes were analyzed after single or concurrent injection of anti-CXCL1, CXCL2, and CCL5 mAbs. The results are illustrated in histograms $\left(\mathrm{J},{ }^{*} \mathrm{P}<0.05\right)$. Each experiment was repeated three times (N=3-4 mice per group). TANs, tumor-associated neutrophils; LLC, Lewis lung carcinoma; RT, radiotherapy; G-CSF, granulocyte colonystimulating factor.

enabled us to investigate the effects of TANs on tumor cells.

\section{RT-recruited neutrophils promote antitumor immunity}

IHC and FC were performed to assess the levels of CTL infiltration in the tumors, spleens, and draining lymph nodes of the mice on day 3 after local irradiation
(Figure $3 A$ and $B$ ). Significant upregulation of CTLs was observed in the RT-treated mice, especially in those treated with concurrent G-CSF, compared with the control mice (all $\mathrm{P}<0.05)$. CTLs accumulated specifically in mice with high neutrophil levels. Furthermore, CTL accumulation was significantly inhibited in the tumors $(\mathrm{P}=0.031)$ and draining lymph nodes $(\mathrm{P}=0.029)$ of neutrophil-depleted mice that 


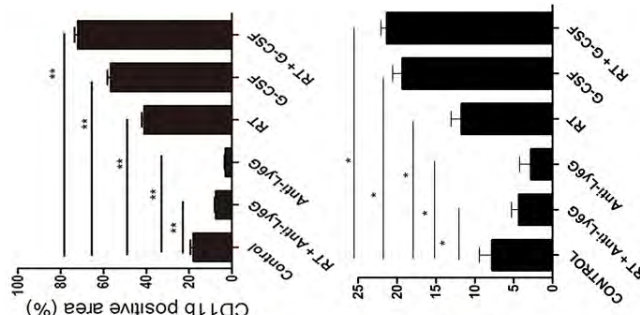

(\%) әбециәлдәd
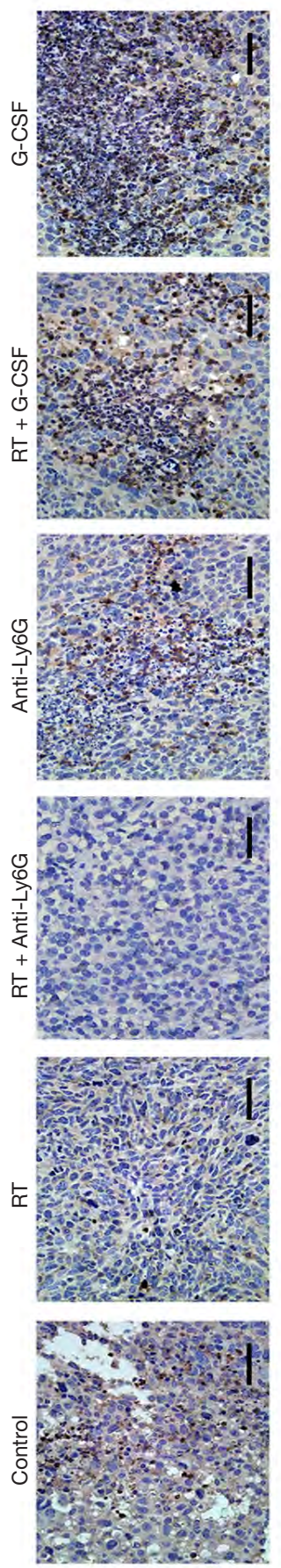

$\varangle$
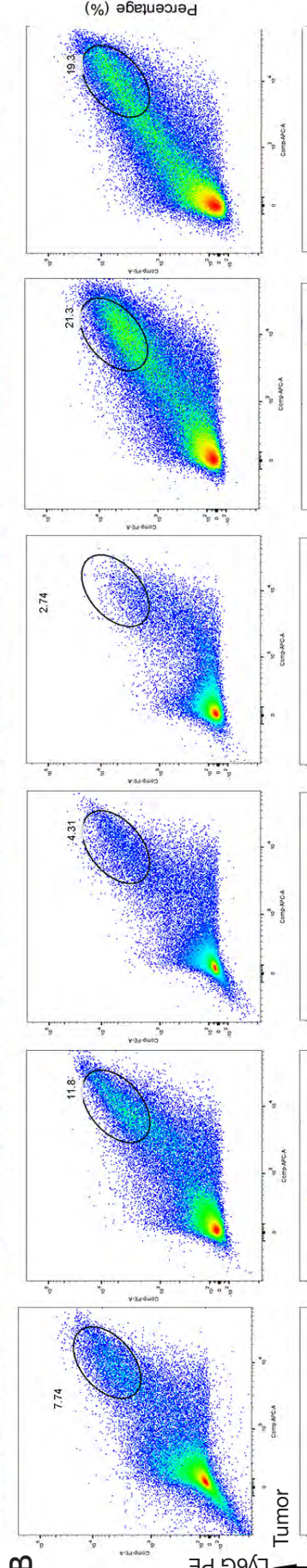

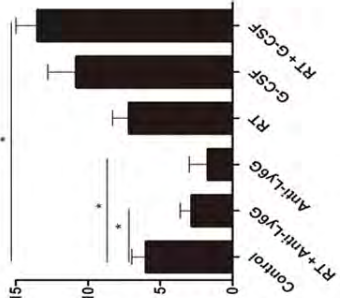

(\%) әбедฺนәวдәd
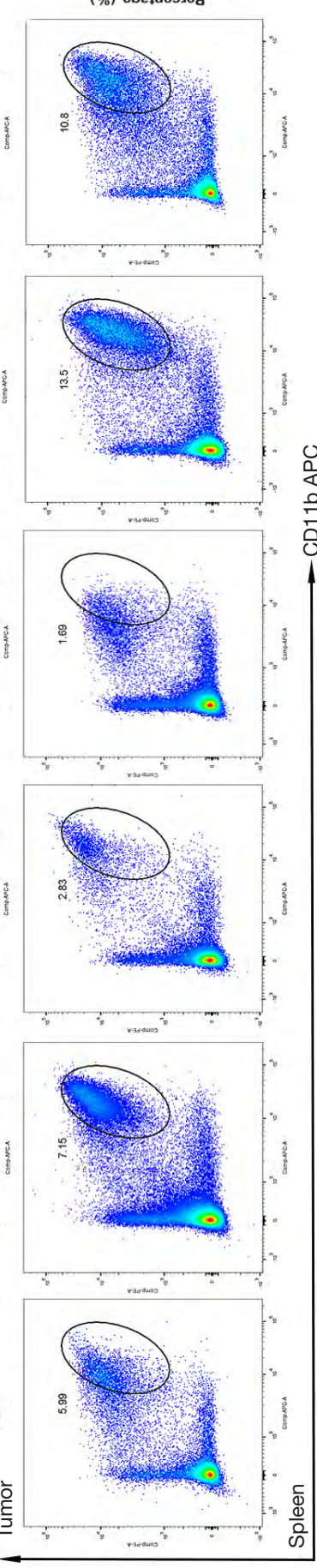

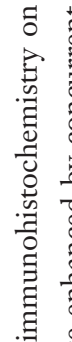

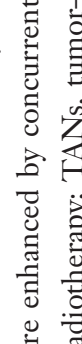

宫苛

ت्ञ

.

党

范

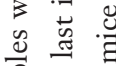

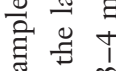

is

要远

至

$\approx$

ํํำ

एँ.

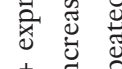

南.

$\overrightarrow{0}$

U.

峁苛 苛

के के

¿ 5

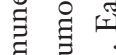

.

品 v

点桴

क 0.

娄

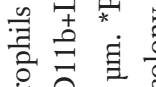

今0

घ

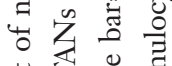

苞苞苟

至定程

है ڤิ

공

원

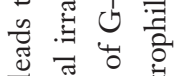

ฮु

두음

$\sim$ 氖苛

必.

总分署 
underwent radiation treatment compared to the control group. Notably, G-CSF administration enhanced CTL infiltration mediated by RT-recruited TANs in the tumors $(\mathrm{P}<0.001)$, spleens $(\mathrm{P}=0.028)$, and draining lymph nodes $(\mathrm{P}=0.033)$.

The levels of granzyme production, which reflect the activation status of CTLs, were also assessed. The levels of granzyme production were observed to be upregulated in mice with high neutrophil levels. This result suggested that the newly recruited neutrophils after RT upregulated the number and enhanced the activation status of CTLs, and G-CSF treatment enhanced this effect (Figure $4 A$, all $\mathrm{P}<0.05)$. In addition, the degree of Treg cell accumulation in the tumor, spleen, and draining lymph nodes of the mice was assessed through FC analysis (Figure 4B). Mice that received concurrent RT treatment and G-CSF subcutaneously injected in proximity to the tumor had significantly fewer Tregs than the control mice in the tumor and spleen. Treg levels were also significantly downregulated in mice with high levels of neutrophil infiltration, whereas anti-Ly6G-mediated neutrophil abrogation following RT led to the upregulation of Treg levels (all $\mathrm{P}<0.05$ ). However, mice treated with RT alone exhibited upregulated Treg levels in lymph nodes $(\mathrm{P}<0.05)$.

\section{RT-associated recruitment of neutrophils promotes mesenchymal-epithelial transition}

To investigate whether RT-recruited TANs could inhibit the epithelial-mesenchymal transition (EMT) process, IHC staining (Figure $5 A, B$ ), and RT-qPCR (Figure 5C) were performed to detect epithelial and mesenchymal markers in LLC tumor-bearing mice. Compared with the control mice, the RT and G-CSF group exhibited an inhibition of EMT with E-cadherin upregulation and vimentin downregulation. These results supported the experimentally described role of new recruited TANs in EMT inhibition in LLC tumor-bearing mice. To further investigate whether MET was initiated by the newly recruited neutrophils, irradiated mice were treated with anti-Ly6G mAb during the 5 days of radiation treatment.

\section{TANs inbibit EMT via the ROS-mediated PI3K/Akt/Snail signaling pathway}

To investigate the detailed mechanisms underlying the antitumor effect of RT and G-CSF treatment, sequencing analysis of tumor cells from the RT + G-CSF and RT groups was performed. Several cancer-associated Kyoto Encyclopedia of Genes and Genomes (KEGG) pathways were identified. Interestingly, the PI3K/Akt signaling pathway was found to be downregulated in the KEGG analyses (Figure 6A,B). The PI3K/AKT signaling pathway plays important roles in EMT and lung cancer metastasis. Western blotting was carried out to examine the effects of TANs on PI3K/Akt/Snail activity in tumor tissues (Figure 6C). As expected, the phosphorylation of PI3K/ Akt was significantly inhibited in mice that received RT and G-CSF concurrently. Also, the expression of Snail was downregulated in the mouse models, as detected by western blotting and RT-qPCR (all $\mathrm{P}<0.05$ ). The quantification of protein expression is shown in Figure 6D. The PI3K/ Akt/Snail signaling pathway was inhibited in the mice treated with RT and G-CSF administration. The RT and neutrophil-depleted group showed upregulation of vimentin and downregulation of E-cadherin (Figure 6C,D, both $\mathrm{P}<0.05)$.

To investigate the correlation between ROS production and the PI3K/Akt/Snail signaling pathway, we depleted ROS with NAC and divided the mice into the following groups: (I) control group, (II) RT group, (III) RT + NAC + anti-Ly6G mAb group, (IV) NAC group, and (V) RT + NAC group. The RT + NAC + Ly6G group was used to confirm that neutrophils mediated the RT-induced recruitment and production of ROS. Western blotting showed that RT + anti-Ly6G + ROS depletion had activated $\mathrm{PI} 3 \mathrm{~K} /$ Akt signaling and MET (Figure $6 E, F$ all $\mathrm{P}<0.05$ ). Greater ROS accumulation was observed in RT-recruited TANs than in the normal neutrophils in non-irradiated tumors $(\mathrm{P}<0.05$, Figure $6 G)$. These results suggested that ROS play a crucial role in the reversal of EMT mediated by TANs via the PI3K/Akt signaling pathway in LLC cells. Therefore, we concluded that newly recruited neutrophils can reverse EMT via the ROS-mediated PI3K-AKT-Snail signaling pathway (Figure 7).

\section{Discussion}

The present study yielded the following conclusions. (I) Concurrent administration of G-CSF and RT improves tumor-specific adaptive immune response, radiosensitivity, and survival, in which newly recruited TANs play indispensable roles. (II) G-CSF and RT recruit TANs based on the roles of CXCL1, CXCL2, and CCL-5 generated by DNA double-strand breaks, and these TANs serve as firstline immune responders. (III) Newly recruited neutrophils 

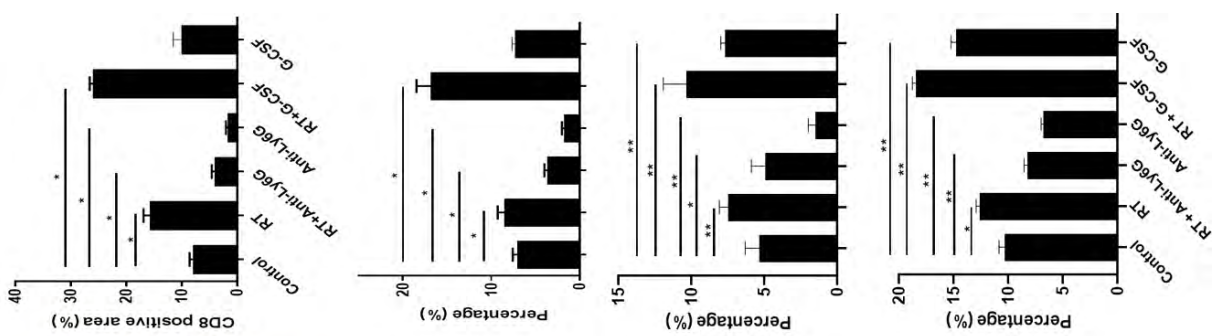

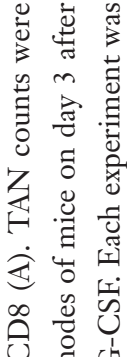
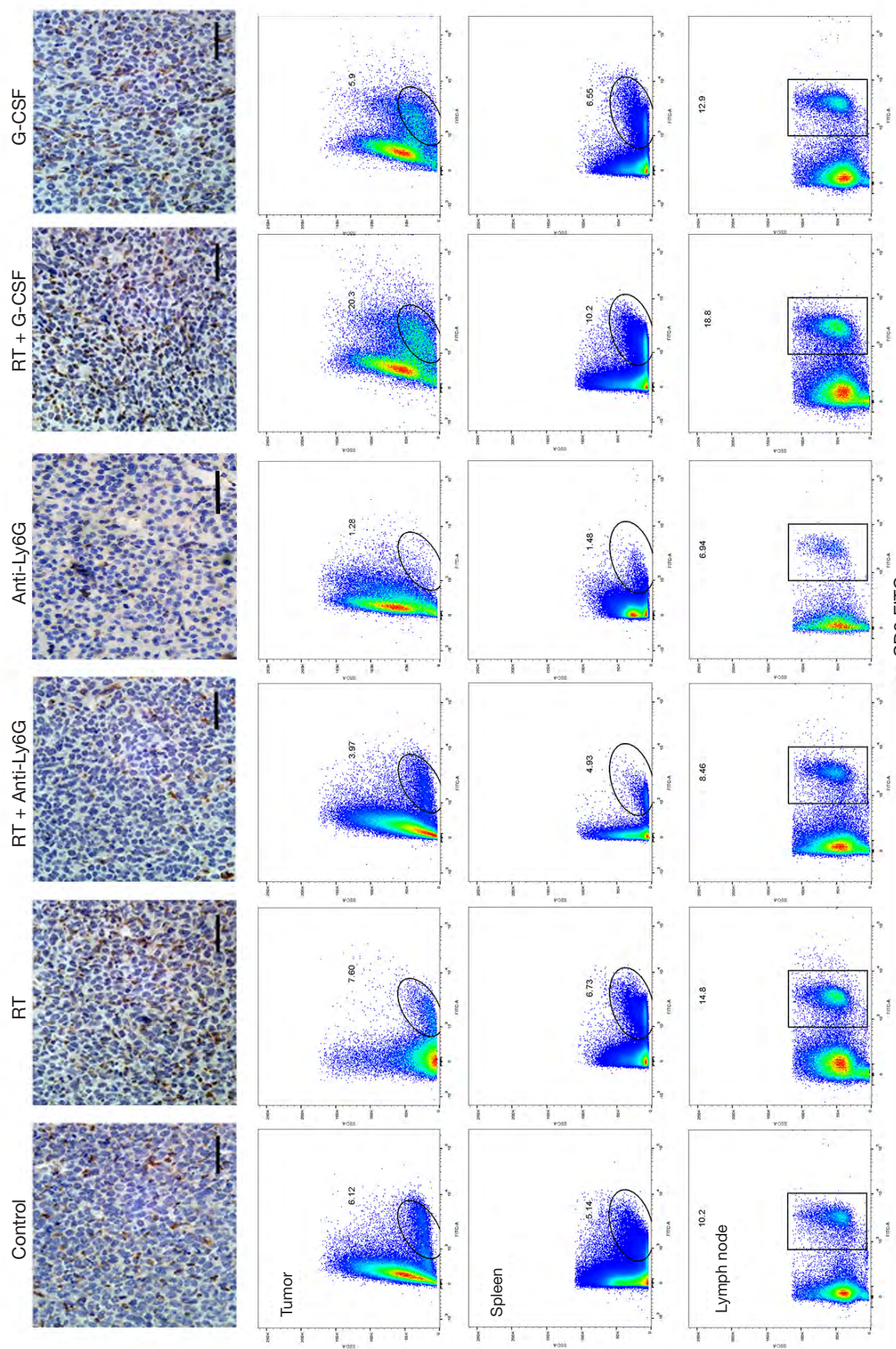

$\varangle$

$\infty$

$\forall$-OSS

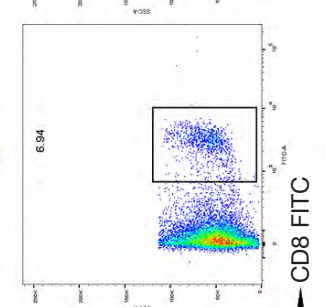

莒

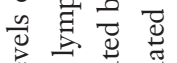

范

.ี.

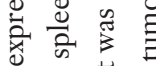

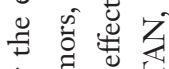

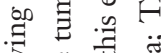

焉

क 青示氞

范

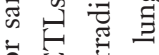

记 $\Xi$

言离总

U

0

㓂芯芯

एँ

产这式

$\simeq \stackrel{0}{\square}$

F

主宁

मे

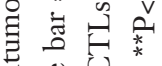

苟U 的

जै

㱐

¿ें छ

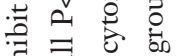

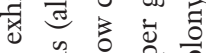

ज ए

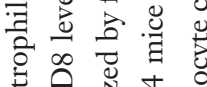

苞芯

专䎡艺苛

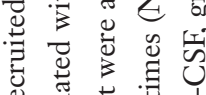

氙苛

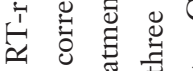

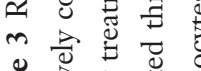

总苟 

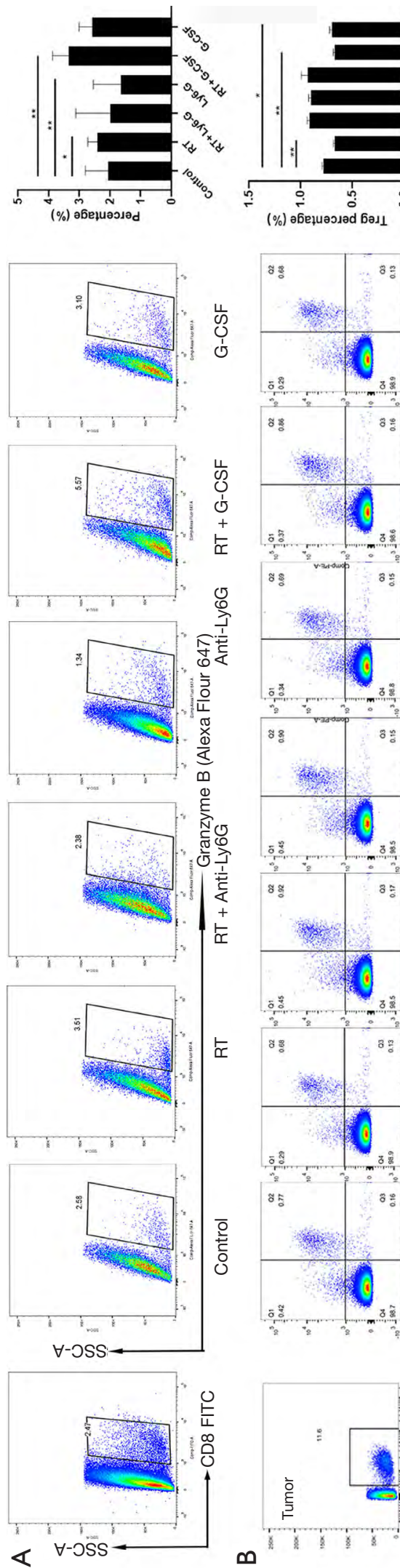
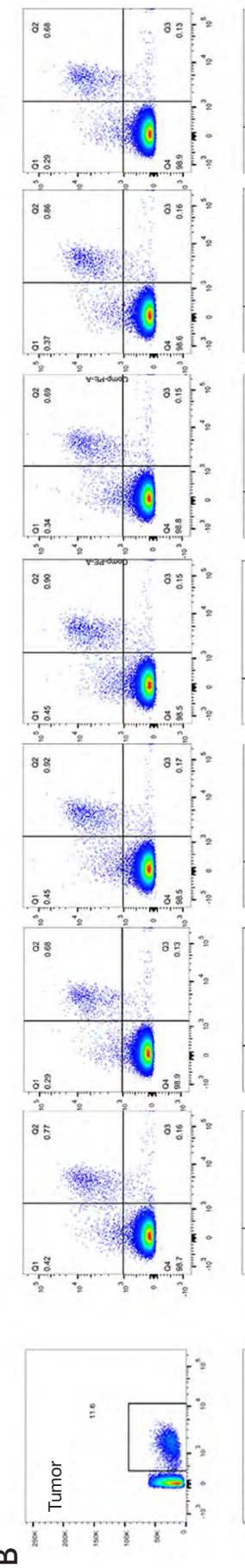
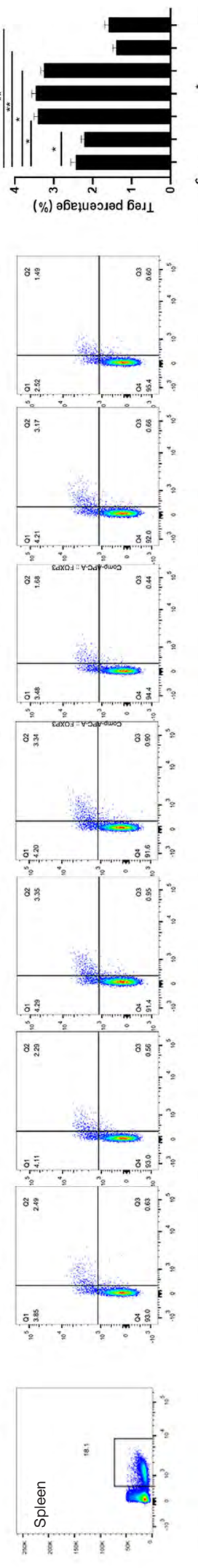

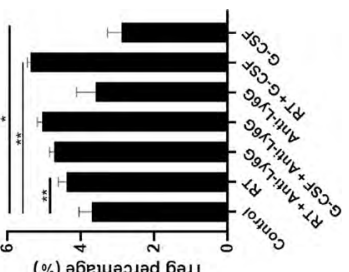

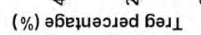
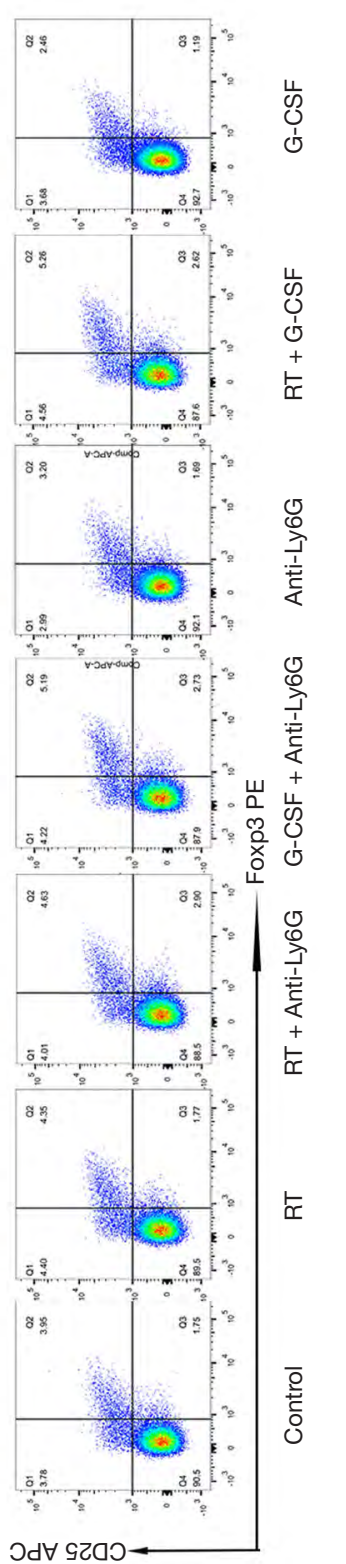

৩d

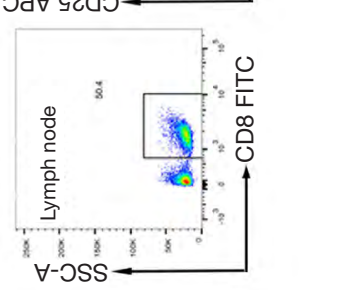

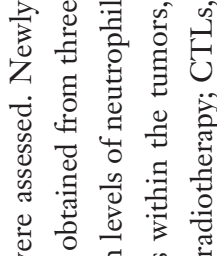

80

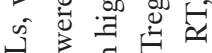

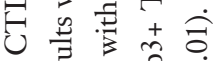

पे

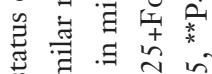

क ज苛苍官

这节去

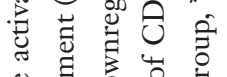

‡ E

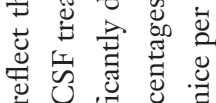

于 b

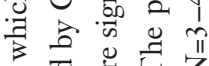

: 可步

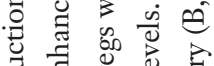

卷苛要

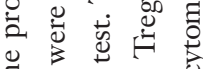

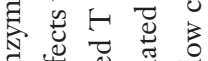

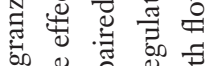

के

足

氙施

E

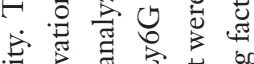

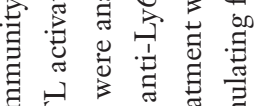

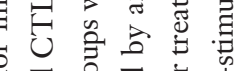

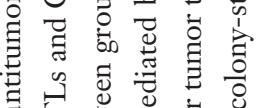

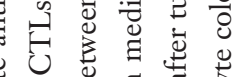

艺记

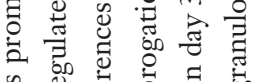

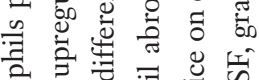

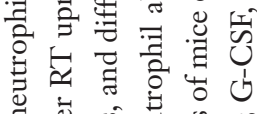

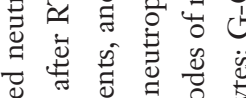

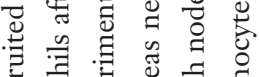

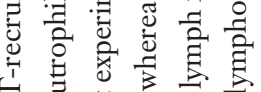

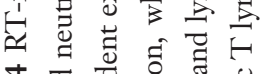

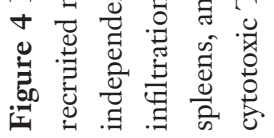




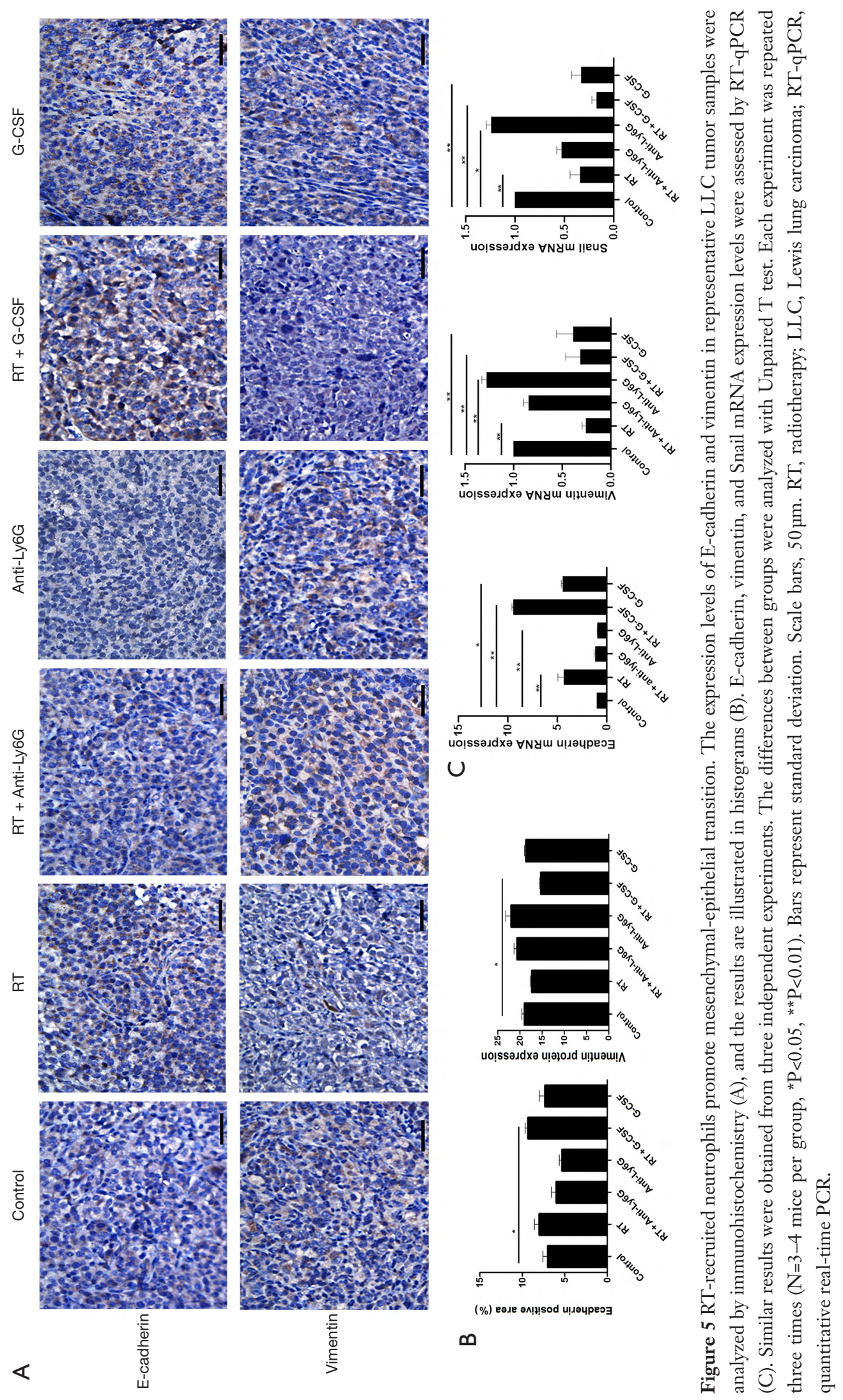



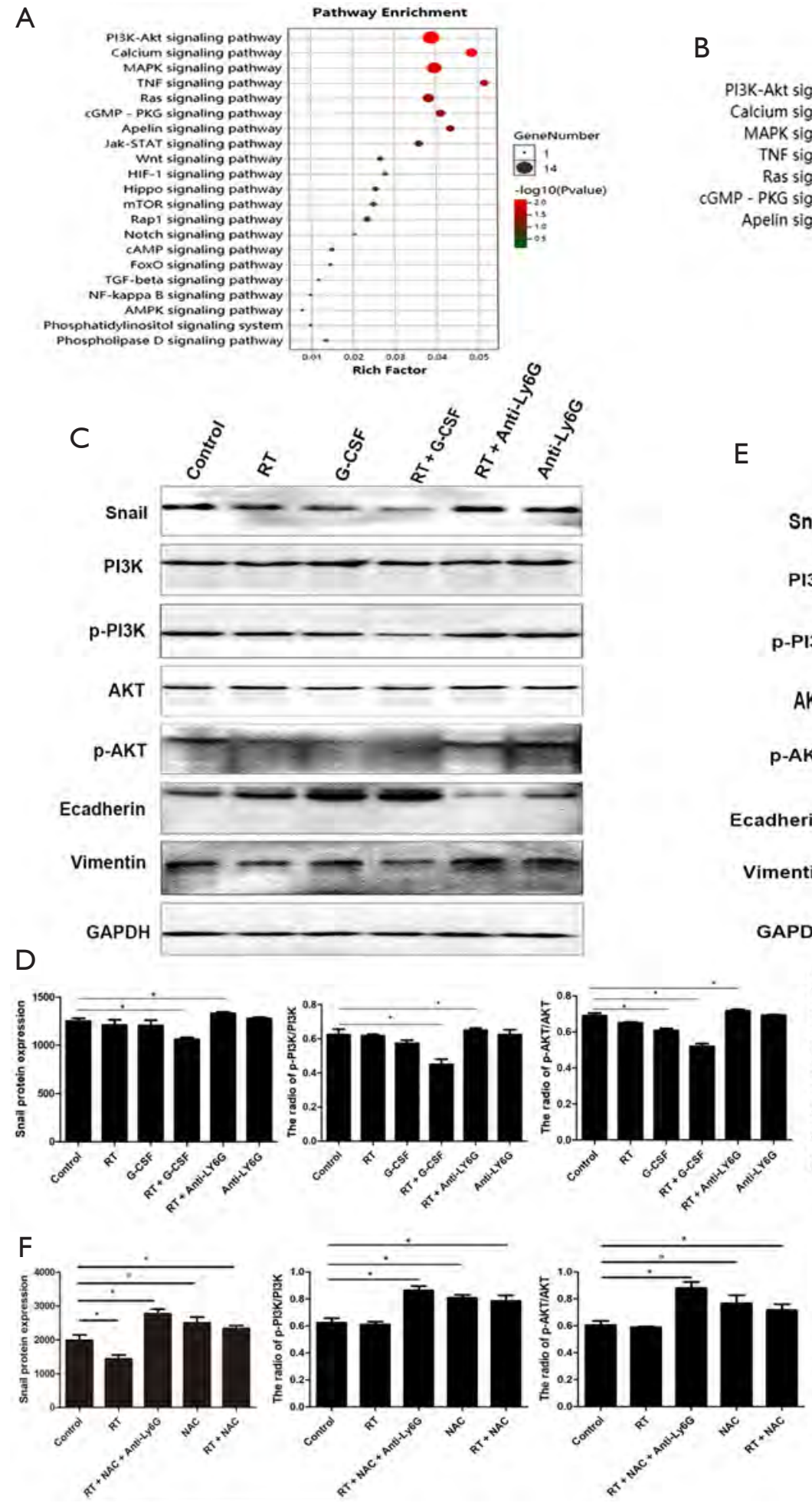
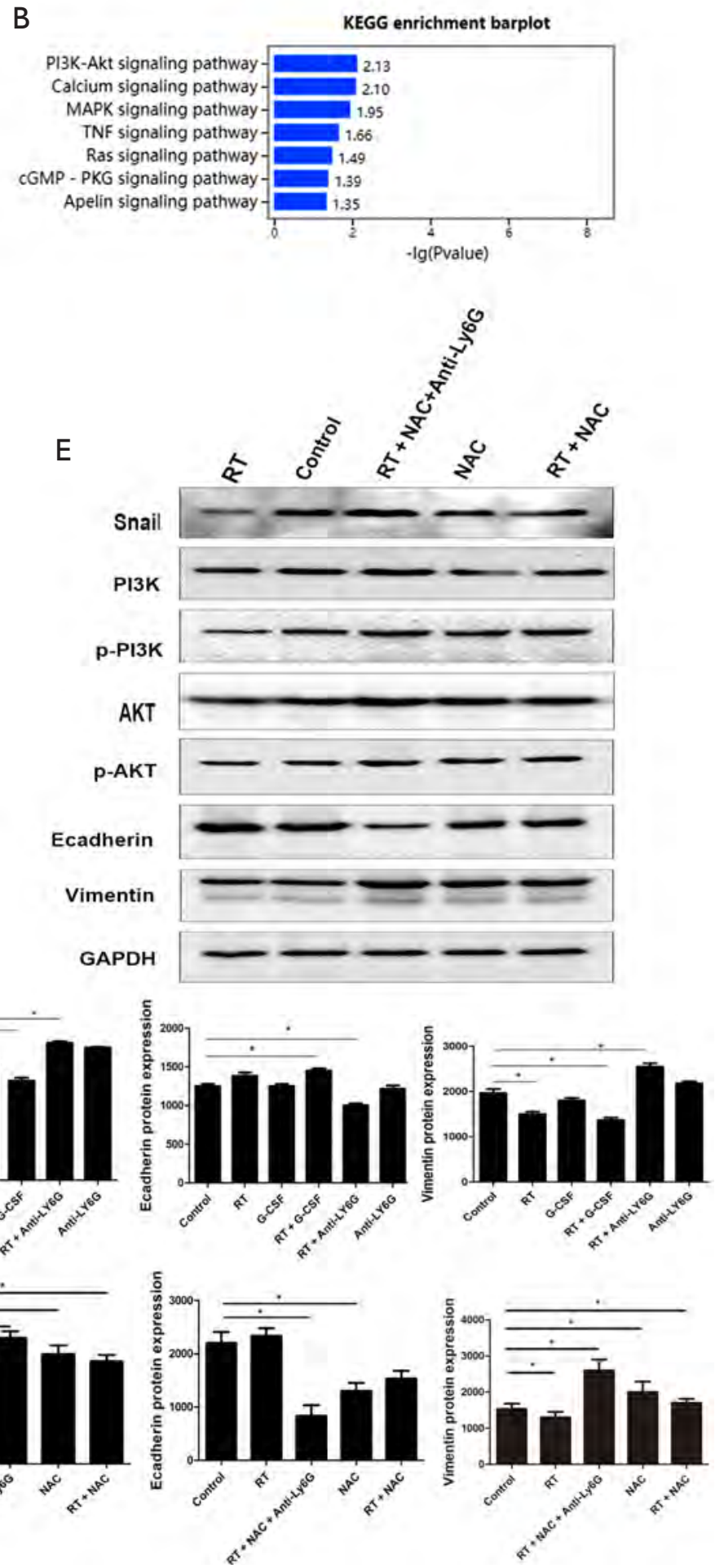

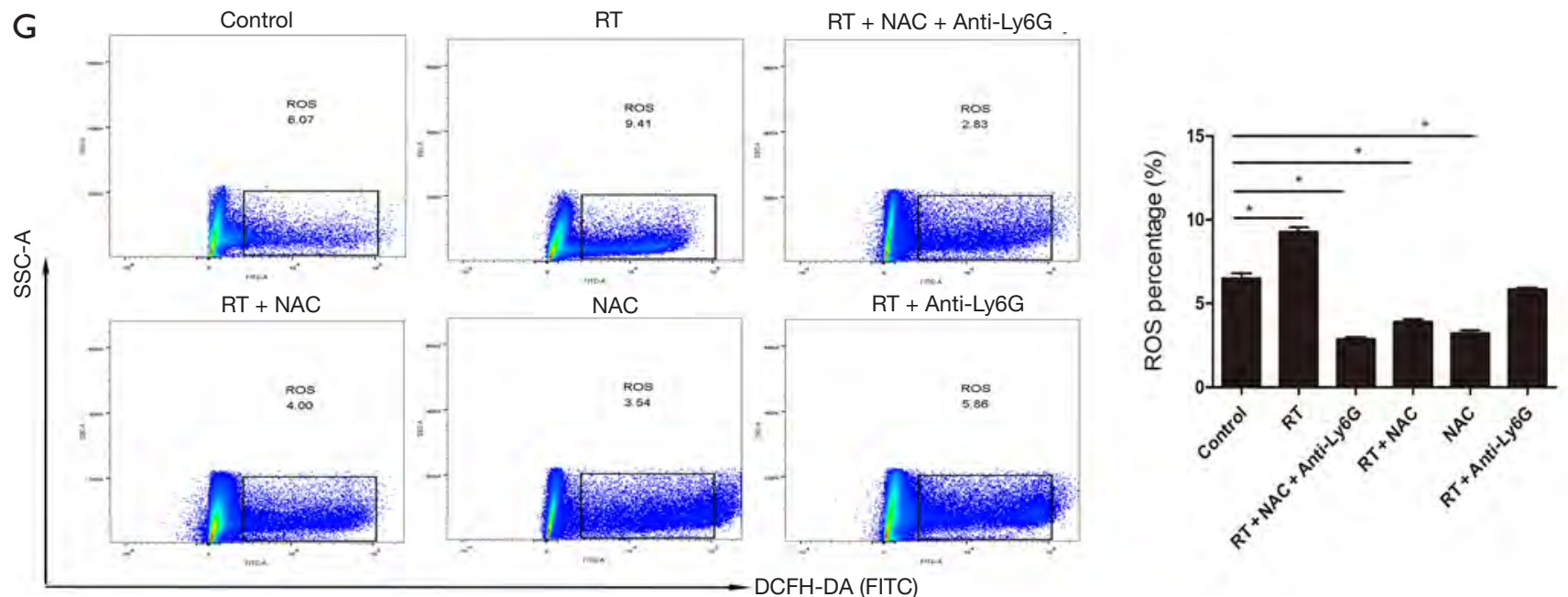

Figure 6 TANs inhibit EMT via the ROS-mediated PI3K/Akt/Snail signaling pathway. KEGG pathway analysis of RNA-sequencing data showed downregulation of the PI3K/Akt signaling pathway (A,B). Western blotting showed that TANs inhibited the activation of PI3K/ Akt signaling as well as the EMT phenotype (C,D). Western blotting showed that mice treated with anti-Ly6G or ROS depletion exhibited activated PI3K/Akt signaling and EMT progression (E,F). Percentage of ROS from mice treated with anti-Ly6G or with ROS depletion, as analyzed by flow cytometry $(\mathrm{G})$. Similar results were obtained from three independent experiments, and differences between groups were analyzed with Unpaired $\mathrm{T}$ test (C, $\mathrm{P}<0.05, \mathrm{~N}=3-4$ mice per group). TANs, tumor-associated neutrophils; KEGG, Kyoto Encyclopedia of Genes and Genomes; EMT, epithelial-mesenchymal transition; ROS, reactive oxygen species.

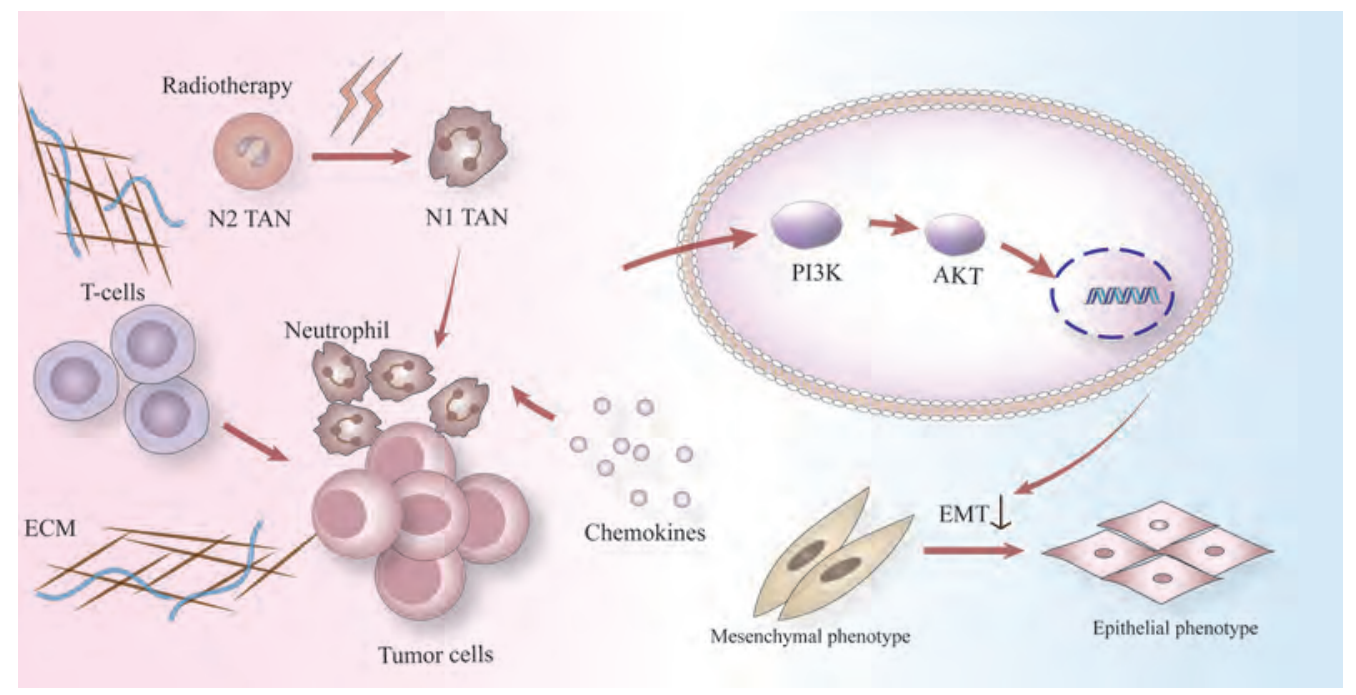

Figure 7 Model of the mechanism of RT-recruited neutrophils in antitumor effect. RT, radiotherapy.

increase ROS expression and inhibit EMT by suppressing the PI3K/Akt/Snail signaling pathway.

Furthermore, compared with patients received a low dose of G-CSF concurrent treatment, a high dose of G-CSF treatment was correlated with significant better OS and PFS in RT-treated lung cancer patients. However, the role of TANs in concurrent treatment of G-CSF and RT was uncertain. We hypothesized that TANs play a major role in concurrent treatment with G-CSF and RT, and we aimed to address the potential effects and mechanisms of TANs in the combination treatment with RT and G-CSF in lung cancer. The present study demonstrated that RT recruits 
TANs to tumor sites, which is consistent with findings reported previously (17). To our knowledge, this is the first study to investigate the mechanism underlying the role of TANs in concurrent treatment with G-CSF and RT LLC tumor-bearing mice and to explore the signaling pathways involved in TAN-mediated antitumor polarization.

The three major innate immunomodulatory cell death patterns induced by non-microbial stimuli-tolerogenic apoptosis, necrosis, and immunogenic apoptosisare denoted as damage-associated molecular patterns (DAMPs) (18). Over in recent years, DAMPs have been considered as the main immunomodulatory pattern of sterile cell death induced by nonmicrobial stimuli, including RT (19). However, neutrophils have been found to recognize pathogenic microbes through the activation of pathogen-associated molecular patterns (PAMPs) by binding to innate pattern recognition receptor (PRRs), thus, resulting in an enhanced immune response against a broad range of pathogens (20). Theoretically, the neutrophils first recruited to the infectious sites serve as the initial innate immune responders that affect functions including phagocytic clearance and direct pathogen elimination (12). In this study, mouse cytokine array analysis showed CXCL1, CXCL2, and CCL5 to be significantly upregulated in LLC cells post-irradiation, as compared with control cells. Moreover, the levels of these chemokines in irradiated LLC cells and the corresponding supernatants were upregulated in a time-dependent manner. The production of CXCL1, CXCL2, and CCL5 is responsible for neutrophil recruitment after irradiation, as observed in several malignant cell lines, in accordance with our findings $(14,21,22)$. Thus, we hypothesized that CXCL1, CXCL2, and CCL5 might have been responsible for the neutrophil recruitment observed in this study. To verify the relationship between these chemokines and neutrophilattracting properties, we blocked cytokines with specific neutralizing antibodies before and during irradiation. The inhibition of cytokines markedly impeded neutrophil recruitment, which demonstrated the absolute neutrophilattracting properties of CXCL1, CXCL2, and CCL5; these cytokines were found to be upregulated and released by tumor cells after irradiation, thereby facilitating neutrophil recruitment. Furthermore, ionizing radiation induces DNA double-stranded breaks (DSBs), which are evidenced by the phosphorylation of histone $2 \mathrm{AX}(\gamma \mathrm{H} 2 \mathrm{AX})(23)$. The expression of $\gamma \mathrm{H} 2 \mathrm{AX}$ in LLC cells was upregulated one day after ionizing radiation treatment, thereby confirming that the DSBs resulted from the RT. Chemokine activation and inflammatory response are initiated in response to severe irreparable DNA damage, particularly DNA DSBs. Therefore, DSBs induce the secretion of inflammatory cytokines and have major biological consequences.

The current study demonstrated that RT-recruited neutrophils play an indispensable role in the induction of the tumor-specific immune response. The number of CTLs was significantly increased in mice receiving RT and/or G-CSF, compared to the control mice. This result demonstrated that the enhanced antitumor immunity was attributable to the upregulation of neutrophil recruitment due to irradiation, an effect that was further enhanced by concurrent G-CSF treatment. These findings agree with those in previous literature illustrating the effects of neutrophils on CTLs. For instance, Radsak et al. reported that polymorphonuclear leukocytes are accessory cells for the activation and amplification of CTLs in an MHC I-dependent manner (24). Additionally, Lim et al. illustrated that tissue-infiltrating neutrophils secrete CXCL12, which acts as a chemotactic signal, resulting in the migration and localization of CTLs to infected sites in the trachea in influenza infection (25). Thus, RT-recruited TANs appear to play a central role in the tumor-specific adaptive immune response mediated by CTLs, which is further enhanced by G-CSF treatment.

The effect of G-CSF on the polarization of TANs appears limited. Immature CD66b+CD10- neutrophils presenting in the circulation of G-CSF-treated donors displayed an immunostimulatory immature morphology, and enhanced CD3/CD28-mediated $\mathrm{T}$ cell proliferation and production of IFN- $\gamma$ (26). A role for G-CSF enhanced RT-mediated antitumor activity by driving formation of N1 neutrophils was reported recently (14).Conversely, some studies identified G-CSF was associated with tumorpromoting neutrophils $(27,28)$. G-CSF facilitates neutrophil proliferation, mobilizes progenitor cells from the bone marrow, induces maturation of immature progenitor cells, and chemoattracts neutrophils to local tissues. It is probably because of the broad functionality of G-CSF, its exact roles in regulating this functional variation have not been definitely explained (29). In addition, radiation regimens may affect the neutrophil phenotypes. An antitumor effect may be more prone to exist in limited fractioned RT (14).

We also investigated the levels of Tregs under the same treatments. Tregs encompass a heterogeneous population of $\mathrm{T}$ cells that exhibit immunosuppressive effects. The results showed that Treg levels were significantly decreased in mice tumor tissue with high levels of neutrophil infiltration 
after RT, particularly those treated with concurrent G-CSF treatment. These results were in accordance with those from a previous study, in which decreased neutrophil levels upregulated Tregs in allergic rhinitis mouse models (30). Furthermore, in this study, neutrophil abrogation mediated by anti-Ly6G upregulated Treg levels. This result, together with the findings on CTLs described above, illustrated that, in addition to direct cytotoxic effects, RT-recruited TANs can exert antitumor effects via the immune system. Notably, the mice that underwent single irradiation exhibited Treg upregulation in lymph nodes. This phenomenon was similar to findings from previous studies in which RT was found to increase the level and enhance the activation status of Treg cells in the TME (31). Owing to apoptosis, necrosis, and programmed cell death of other cells, the percentage of Tregs was upregulated in lymph nodes in our study. Together, these results show that irradiation regulates the immune profile of the TME, resulting in transient recruitment of antitumor TANs to tumor sites.

EMT is an indispensable process during cancer progression and metastasis. This phenomenon can be observed in most cancers in the processes of in situ, malignant tumor cell invasion of surrounding tissues and distant organ metastasis. Recent studies found EMT to be associated with resistance to antitumor therapies, including chemotherapeutic drugs and radiation (32-34). Gomez-Casal $\mathrm{R}$ et al. illustrated that cancer cells that survive RT show an EMT phenotype, including repression of E-cadherin, and upregulation of $\mathrm{N}$-cadherin and vimentin (35). Furthermore, tumor progression is significantly impeded when the reversal of the EMT process is promoted (36). In the present study, we found that RT-recruited neutrophils reversed the EMT in irradiated mouse models, particularly in those treated with concurrent G-CSF administration. These data indicated that TANs trigger MET in LLC cells and consequently improve tumor radiosensitivity in mice. Furthermore, the E-cadherin promoter is suppressed by Snail, which disrupts the epithelial polarity of cells and exhibits mesenchymal phenotypes $(37,38)$. Snail acts as a transcriptional repressor by binding the E-box motifs in the E-cadherin promoter gene $(C D H 1)$ via zinc fingers, which results in EMT progression (39). In 2011, Hung et al. discovered that the upregulation of Snail expression is associated with resistance to antitumor therapy and results in tumor recurrence, metastasis, and poor prognosis in various human cancers (40). In the present study, western blotting showed that Snail expression was significantly downregulated in the RT mice, particularly in those that received concurrent G-CSF treatment. This phenomenon was further confirmed in paraffin-embedded tumor sections subjected to IHC staining. The results suggested that Snail plays a crucial role in the reversal of TAN-mediated EMT, which is in line with previous observations $(40,41)$.

The PI3K/Akt-dependent signaling pathway plays a major role in EMT. Chang et al. indicated that radioresistance in prostate cancer is associated with the activation of the PI3K/Akt signaling pathway (42). Western blotting revealed dephosphorylation of PI3K/Akt in mice that had high levels of neutrophils that were recruited by RT or upregulated by G-CSF treatment. These results confirmed that the TAN-mediated MET occurred via the PI3K/Akt signaling pathway in LLC cells. Furthermore, several investigations have revealed that ROS generation is an event upstream of the PI3K/Akt pathway. ROS, which comprise various short-lived and oxygen-containing molecules, can affect physiological processes and activate multiple intracellular signaling pathways by regulating the kinase activity and phosphorylation of proteins via cysteine oxidation (43-45). In the present study, we found that RTrecruited TANs generated significantly more ROS than normal neutrophils. Zaragoza-Campillo et al. discovered that ROS treatment leads to Akt inactivation in a timedependent manner (46). Li et al. demonstrated the essential role of ROS in Akt dephosphorylation (47). Some studies have also shown that high-glucose exposure efficiently decreases ROS levels and alters dephosphorylation in the PI3K/Akt pathway (48). Our analytical results suggested that RT-recruited TANs enhance ROS generation, which in turn inactivates the effects on the PI3K/Akt signaling pathway. Besides, ROS was depleted with NAC to investigate the correlation between ROS production and the PI3K/Akt/Snail signaling pathway. Snail expression and EMT progression were significantly upregulated in NAC-treated mice. We also observed that phosphorylation of PI3K/Akt was upregulated in mice treated with NAC, compared with the control mice. These results suggested that ROS plays a crucial role in the reversal of TANmediated EMT via the PI3K/Akt signaling pathway in LLC cells. Moreover, the effects of TANs on tumors were enhanced by concurrent G-CSF treatment. Thus, we conclude that TANs can reverse EMT via the ROSmediated PI3K-AKT-Snail signaling pathway.

Previous research showed that prophylactic G-CSF resulted in an unexpected reduced local control and should be given in radiotherapy regimen only with strong hematologic indication (49). Other researches also showed 
that simultaneous treatment with G-CSF during RT would reduce the mobilization of CD34+ progenitor cells and exhaust the bone marrow capacity, as well as increase the risk of thrombocytopenia $(50,51)$. Thus, CSFs should be avoided in patients receiving concomitant chemotherapy and radiation therapy, particularly involving the mediastinum. In the absence of chemotherapy, therapeutic use of CSFs may be considered in patients receiving radiation therapy alone if prolonged delays secondary to neutropenia are expected. However, though there were concerns regarding the safety and efficacy of combination treatment of G-CSF and CCRT, Benna et al. also pointed out that no abnormal infield event was reported in patients received CSFs-chemoradiation so far (52). G-CSF could be a novel therapeutic target to mitigate the harmful effect of radiotherapy including neutropenia for the treatment of NSCLC (53).

Our conclusions were based on animal studies, which helped to provide useful refinements in the methodology of animal experiments about G-CSF in combination with radiotherapy. The limitation of preclinical animal studies is that some caution is warranted when extrapolating experimental results to the clinic. Pre-clinical data from in vitro and in vivo studies does not perfectly mimic human pathophysiology. Thus, further investigation to elucidate the role of G-CSF in combination with radiotherapy were needed.

The in vitro and in vivo results of this study confirmed that RT leads to recruitment of neutrophils through the co-release of CXCL1, CXCL2, and CCL5. RT-recruited TANs induce MET via the inhibition of the ROS-mediated PI3K/Akt/Snail signaling pathway, thus, improving antitumor immune activity. This effect is further enhanced by concurrent G-CSF treatment. In summary, newly recruited TANs are a major contributor to the antitumor effects of concurrent RT and G-CSF, and this mechanism may serve as a novel antitumor therapeutic strategy for patients with cancer.

\section{Acknowledgments}

The authors appreciate the academic support from AME Radiotherapy Collaborative Group.

Funding: This study was supported by grants from the National Natural Science Foundation of China (grant No. 81672974, No. 81602719, and No. 81803043) and the Science and Technology Development Plan of Shandong Province (No. 2017GSF18111). This study was supported by the Munich Excellence Training Initiative for Physician Scientists (Metiphys to LK).

\section{Footnote}

Reporting Checklist: The authors have completed the ARRIVE reporting checklist. Available at http://dx.doi. org/10.21037/tlcr-21-152

Data Sharing Statement: Available at http://dx.doi. org/10.21037/tlcr-21-152

Conflicts of Interest: All authors have completed the ICMJE uniform disclosure form (available at http://dx.doi. org/10.21037/tlcr-21-152). Dr. Rades reports grants from MerckSerono, personal fees from Elsevier, outside the submitted work. The other authors have no conflicts of interest to declare.

Ethical Statement: The authors are accountable for all aspects of the work in ensuring that questions related to the accuracy or integrity of any part of the work are appropriately investigated and resolved. All animal experiments conducted were reviewed and approved by the Institutional Animal Care and Use Committee of Shandong Provincial Qianfoshan Hospital (no. 2016-S017). Experiments were performed in compliance with the Guide for Care and Use of Laboratory Animals (NIH publication no. 85-23, revised 1996).

Open Access Statement: This is an Open Access article distributed in accordance with the Creative Commons Attribution-NonCommercial-NoDerivs 4.0 International License (CC BY-NC-ND 4.0), which permits the noncommercial replication and distribution of the article with the strict proviso that no changes or edits are made and the original work is properly cited (including links to both the formal publication through the relevant DOI and the license). See: https://creativecommons.org/licenses/by-nc-nd/4.0/.

\section{References}

1. Schneider BJ, Daly ME, Kennedy EB, et al. Stereotactic Body Radiotherapy for Early-Stage Non-Small-Cell Lung Cancer: American Society of Clinical Oncology Endorsement of the American Society for Radiation Oncology Evidence-Based Guideline. J Clin Oncol 2018;36:710-9. 
2. Hansen RN, Zhang Y, Seal B, et al. Long-term survival trends in patients with unresectable stage III non-small cell lung cancer receiving chemotherapy and radiation therapy: a SEER cancer registry analysis. BMC Cancer 2020;20:276.

3. Roos WP, Thomas AD, Kaina B. DNA damage and the balance between survival and death in cancer biology. Nat Rev Cancer 2016;16:20-33.

4. Ozpiskin OM, Zhang L, Li JJ. Immune targets in the tumor microenvironment treated by radiotherapy. Theranostics 2019;9:1215-31.

5. Jiang W, Chan CK, Weissman IL, et al. Immune Priming of the Tumor Microenvironment by Radiation. Trends Cancer 2016;2:638-45.

6. Delgado O, Batten KG, Richardson JA, et al. Radiationenhanced lung cancer progression in a transgenic mouse model of lung cancer is predictive of outcomes in human lung and breast cancer. Clin Cancer Res 2014;20:1610-22.

7. Dongre A, Weinberg RA. New insights into the mechanisms of epithelial-mesenchymal transition and implications for cancer. Nat Rev Mol Cell Biol 2019;20:69-84.

8. Hinshaw DC, Shevde LA. The Tumor Microenvironment Innately Modulates Cancer Progression. Cancer Res 2019;79:4557-66.

9. Fridlender ZG, Sun J, Kim S, et al. Polarization of tumorassociated neutrophil phenotype by TGF-beta: "N1" versus "N2" TAN. Cancer Cell 2009;16:183-94.

10. Jaillon S, Ponzetta A, Di Mitri D, et al. Neutrophil diversity and plasticity in tumour progression and therapy. Nat Rev Cancer 2020;20:485-503.

11. Jablonska J, Leschner S, Westphal K, et al. Neutrophils responsive to endogenous IFN-beta regulate tumor angiogenesis and growth in a mouse tumor model. J Clin Invest 2010;120:1151-64.

12. Garg AD, Vandenberk L, Fang S, et al. Pathogen responselike recruitment and activation of neutrophils by sterile immunogenic dying cells drives neutrophil-mediated residual cell killing. Cell Death Differ 2017;24:832-43.

13. Rapoport AP, Abboud CN, DiPersio JF. Granulocytemacrophage colony-stimulating factor (GM-CSF) and granulocyte colony-stimulating factor (G-CSF): receptor biology, signal transduction, and neutrophil activation. Blood Rev 1992;6:43-57.

14. Takeshima T, Pop LM, Laine A, et al. Key role for neutrophils in radiation-induced antitumor immune responses: Potentiation with G-CSF. Proc Natl Acad Sci U S A 2016;113:11300-5.
15. Frazer LC, O'Connell CM, Andrews CW, Jr., et al. Enhanced neutrophil longevity and recruitment contribute to the severity of oviduct pathology during Chlamydia muridarum infection. Infect Immun 2011;79:4029-41.

16. Rodier F, Coppe JP, Patil CK, et al. Persistent DNA damage signalling triggers senescence-associated inflammatory cytokine secretion. Nat Cell Biol 2009;11:973-9.

17. Lorimore SA, Coates PJ, Scobie GE, et al. Inflammatorytype responses after exposure to ionizing radiation in vivo: a mechanism for radiation-induced bystander effects? Oncogene 2001;20:7085-95.

18. Garg AD, Galluzzi L, Apetoh L, et al. Molecular and Translational Classifications of DAMPs in Immunogenic Cell Death. Front Immunol 2015;6:588.

19. Zitvogel L, Kepp O, Kroemer G. Decoding cell death signals in inflammation and immunity. Cell 2010;140:798-804.

20. Durr MC, Kristian SA, Otto M, et al. Neutrophil chemotaxis by pathogen-associated molecular patterns-formylated peptides are crucial but not the sole neutrophil attractants produced by Staphylococcus aureus. Cell Microbiol 2006;8:207-17.

21. Torres IM, Patankar YR, Berwin B. Acidosis exacerbates in vivo IL-1-dependent inflammatory responses and neutrophil recruitment during pulmonary Pseudomonas aeruginosa infection. Am J Physiol Lung Cell Mol Physiol 2018;314:L225-35.

22. Dunn JLM, Kartchner LB, Stepp WH, et al. Blocking CXCL1-dependent neutrophil recruitment prevents immune damage and reduces pulmonary bacterial infection after inhalation injury. Am J Physiol Lung Cell Mol Physiol 2018;314:L822-34.

23. Harding SM, Benci JL, Irianto J, et al. Mitotic progression following DNA damage enables pattern recognition within micronuclei. Nature 2017;548:466-70.

24. Radsak M, Iking-Konert C, Stegmaier S, et al. Polymorphonuclear neutrophils as accessory cells for T-cell activation: major histocompatibility complex class II restricted antigen-dependent induction of T-cell proliferation. Immunology 2000;101:521-30.

25. Lim K, Hyun YM, Lambert-Emo K, et al. Neutrophil trails guide influenza-specific CD8(+) T cells in the airways. Science 2015;349:aaa4352.

26. Marini O, Costa S, Bevilacqua D, et al. Mature CD10(+) and immature CD10(-) neutrophils present in G-CSFtreated donors display opposite effects on T cells. Blood 2017;129:1343-56. 
27. Kowanetz M, Wu X, Lee J, et al. Granulocyte-colony stimulating factor promotes lung metastasis through mobilization of Ly6G+Ly6C+ granulocytes. Proc Natl Acad Sci U S A 2010;107:21248-55.

28. Waight JD, Hu Q, Miller A, et al. Tumor-derived G-CSF facilitates neoplastic growth through a granulocytic myeloid-derived suppressor cell-dependent mechanism. PLoS One 2011;6:e27690.

29. Mouchemore KA, Anderson RL, Hamilton JA. Neutrophils, G-CSF and their contribution to breast cancer metastasis. FEBS J 2018;285:665-79.

30. Gu ZW, Wang YX, Cao ZW. Neutralization of interleukin-17 suppresses allergic rhinitis symptoms by downregulating Th2 and Th17 responses and upregulating the Treg response. Oncotarget 2017;8:22361-9.

31. Muroyama Y, Nirschl TR, Kochel CM, et al. Stereotactic Radiotherapy Increases Functionally Suppressive Regulatory T Cells in the Tumor Microenvironment. Cancer Immunol Res 2017;5:992-1004.

32. Kong D, Li Y, Wang Z, et al. Cancer Stem Cells and Epithelial-to-Mesenchymal Transition (EMT)-Phenotypic Cells: Are They Cousins or Twins? Cancers (Basel) 2011;3:716-29.

33. Chang L, Graham PH, Hao J, et al. Emerging roles of radioresistance in prostate cancer metastasis and radiation therapy. Cancer Metastasis Rev 2014;33:469-96.

34. Singh A, Settleman J. EMT, cancer stem cells and drug resistance: an emerging axis of evil in the war on cancer. Oncogene 2010;29:4741-51.

35. Gomez-Casal R, Bhattacharya C, Ganesh N, et al. Nonsmall cell lung cancer cells survived ionizing radiation treatment display cancer stem cell and epithelialmesenchymal transition phenotypes. Mol Cancer 2013;12:94.

36. Galvan JA, Gonzalez MV, Crespo G, et al. Snail nuclear expression parallels higher malignancy potential in neuroendocrine lung tumors. Lung Cancer 2010;69:289-95.

37. Thiery JP, Lim CT. Tumor dissemination: an EMT affair. Cancer Cell 2013;23:272-3.

38. Zhang P, Sun Y, Ma L. ZEB1: at the crossroads of epithelial-mesenchymal transition, metastasis and therapy resistance. Cell Cycle 2015;14:481-7.

39. Cano A, Perez-Moreno MA, Rodrigo I, et al. The transcription factor snail controls epithelial-mesenchymal transitions by repressing E-cadherin expression. Nat Cell Biol 2000;2:76-83.
40. Hung JJ, Yang MH, Hsu HS, et al. Prognostic significance of hypoxia-inducible factor-1alpha, TWIST1 and Snail expression in resectable non-small cell lung cancer. Thorax 2009;64:1082-9.

41. Lamouille S, Xu J, Derynck R. Molecular mechanisms of epithelial-mesenchymal transition. Nat Rev Mol Cell Biol 2014;15:178-96.

42. Chang L, Graham PH, Hao J, et al. Acquisition of epithelial-mesenchymal transition and cancer stem cell phenotypes is associated with activation of the PI3K/Akt/ mTOR pathway in prostate cancer radioresistance. Cell Death Dis 2013;4:e875.

43. Droge W. Free radicals in the physiological control of cell function. Physiol Rev 2002;82:47-95.

44. Pourova J, Kottova M, Voprsalova M, et al. Reactive oxygen and nitrogen species in normal physiological processes. Acta Physiol (Oxf) 2010;198:15-35.

45. Kamata H, Hirata H. Redox regulation of cellular signalling. Cell Signal 1999;11:1-14.

46. Zaragoza-Campillo MA, Moran J. Reactive Oxygen Species Evoked by Potassium Deprivation and Staurosporine Inactivate Akt and Induce the Expression of TXNIP in Cerebellar Granule Neurons. Oxid Med Cell Longev 2017;2017:8930406.

47. Li F, Li Q, Shi X, et al. Maslinic acid inhibits impairment of endothelial functions induced by high glucose in HAEC cells through improving insulin signaling and oxidative stress. Biomed Pharmacother 2017;95:904-13.

48. Ho FM, Lin WW, Chen BC, et al. High glucose-induced apoptosis in human vascular endothelial cells is mediated through NF-kappaB and c-Jun NH2-terminal kinase pathway and prevented by PI3K/Akt/eNOS pathway. Cell Signal 2006;18:391-9.

49. Staar S, Rudat V, Stuetzer H, et al. Intensified hyperfractionated accelerated radiotherapy limits the additional benefit of simultaneous chemotherapy--results of a multicentric randomized German trial in advanced head-and-neck cancer. Int J Radiat Oncol Biol Phys 2001;50:1161-71.

50. Pape H, Orth K, Heese A, et al. G-CSF during large field radiotherapy reduces bone marrow recovery capacity. Eur J Med Res 2006;11:322-8.

51. Sheikh H, Colaco R, Lorigan P, et al. Use of G-CSF during concurrent chemotherapy and thoracic radiotherapy in patients with limited-stage small-cell lung cancer safety data from a phase II trial. Lung Cancer 2011;74:75-9.

52. Benna M, Guy JB, Bosacki C, et al. Chemoradiation and 
granulocyte-colony or granulocyte macrophage-colony stimulating factors (G-CSF or GM-CSF): time to think out of the box? Br J Radiol 2020;93:20190147.

53. Cui YH, Suh Y, Lee HJ, et al. Radiation promotes

Cite this article as: Liu Q, Hao Y, Du R, Hu D, Xie J, Zhang J, Deng G, Liang N, Tian T, Käsmann L, Rades D, Rim CH, Hu P, Zhang J. Radiotherapy programs neutrophils to an antitumor phenotype by inducing mesenchymal-epithelial transition. Transl Lung Cancer Res 2021;10(3):1424-1443. doi: 10.21037/ tlcr-21-152 invasiveness of non-small-cell lung cancer cells through granulocyte-colony-stimulating factor. Oncogene 2015;34:5372-82. 
Supplementary

Table S1 Primary antibodies used in the study

\begin{tabular}{|c|c|c|}
\hline Antibody & Use & Company \\
\hline CXCL1 & 50 ng/body, blocking & $R \& D$ Systems \\
\hline CXCL2 & 50 ng/body, blocking & R\&D Systems \\
\hline CCL5 & 50 ng/body, blocking & $R \& D$ Systems \\
\hline CD11b & 1:200 for IHC & Abcam \\
\hline Vimentin & $1: 300, \mathrm{IHC}$ & Cell Signaling Technology \\
\hline E-cadherin & 1:300, IHC & Cell Signaling Technology \\
\hline CD11b-APC & $1: 20, \mathrm{FC}$ & BioLegend \\
\hline Granzyme B antibody-Alexa Fluor 647 & $1: 20, \mathrm{FC}$ & Biolegend \\
\hline CD4-FITC & $1: 20, \mathrm{FC}$ & eBioscience \\
\hline CD25-APC & $1: 20, \mathrm{FC}$ & eBioscience \\
\hline FoxP3-PE & $1: 20, \mathrm{FC}$ & eBioscience \\
\hline $\mathrm{N}$-acetylcysteine & $200 \mathrm{mg} / \mathrm{kg}$, ROS depletion & Beyotime \\
\hline E-cadherin & 1:1000, Wb & Cell Signaling Technology \\
\hline Vimentin & 1:1000, Wb & Cell Signaling Technology \\
\hline Snail & $1: 1000, W b$ & Cell Signaling Technology \\
\hline GAPDH & $1: 1000, W b$ & Proteintech \\
\hline
\end{tabular}

WB, western blotting; IHC, immunohistochemistry; FC, flow cytometry. 


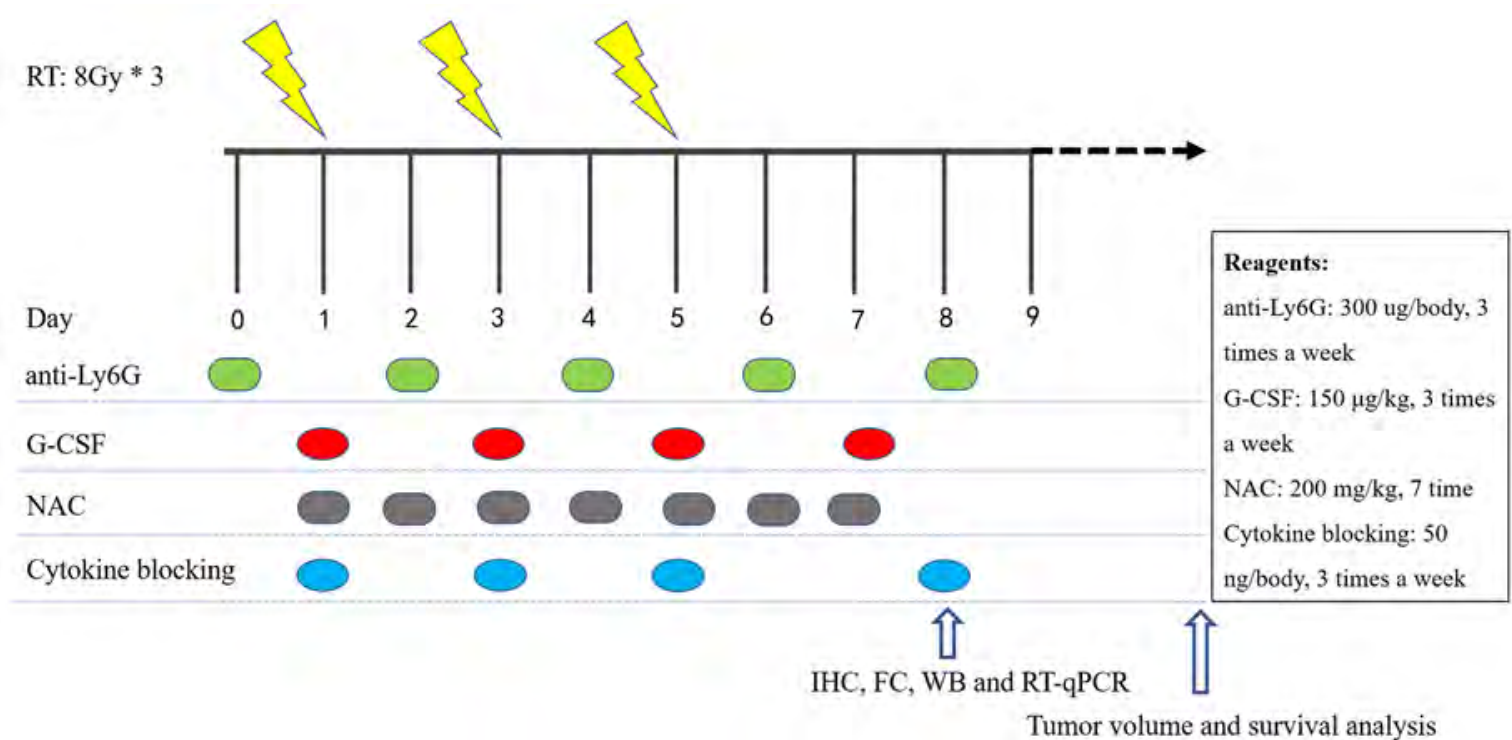

Figure S1 A time-line diagram for study design. RT, radiotherapy; G-CSF, granulocyte colony-stimulating factor; NAC, N-acetylcysteine; IHC, immunohistochemistry; FC, flow cytometry; WB, western blotting; RT-qPCR, quantitative real-time PCR.
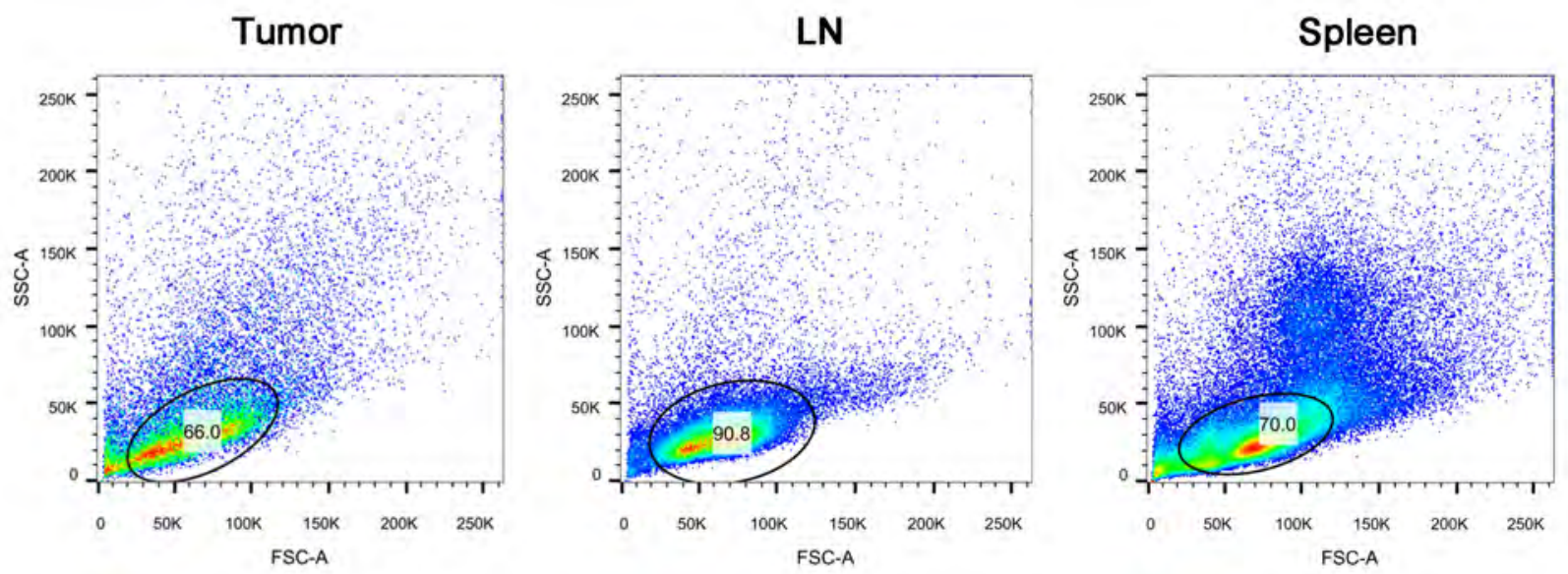

Figure S2 The gating strategy and representative flow cytometry. In total, 100,000 events were recorded, and were initially gated according to FSC/SSC profiles. 